\title{
riskRegression: Predicting the Risk of an Event using Cox Regression Models
}

by Brice Ozenne, Anne Lyngholm Sørensen, Thomas Scheike, Christian Torp-Pedersen, Thomas Alexander Gerds

\begin{abstract}
In the presence of competing risks a prediction of the time-dynamic absolute risk of an event can be based on cause-specific Cox regression models for the event and the competing risks (Benichou and Gail, 1990). We present computationally fast and memory optimized C++ functions with an R interface for predicting the covariate specific absolute risks, their confidence intervals, and their confidence bands based on right censored time to event data. We provide explicit formulas for our implementation of the estimator of the (stratified) baseline hazard function in the presence of tied event times. As a by-product we obtain fast access to the baseline hazards (compared to survival: : basehaz()) and predictions of survival probabilities, their confidence intervals and confidence bands. Confidence intervals and confidence bands are based on point-wise asymptotic expansions of the corresponding statistical functionals. The software presented here is implemented in the riskRegression package.
\end{abstract}

\section{Introduction}

Predictions of hazards and risks based on a Cox regression analysis need to be fast and memory efficient, especially in large data, in simulation studies, and for cross-validation or bootstrap loops. The CRAN task view Survival lists many $R$ packages implementing the Cox regression model and extensions thereof. Among the most popular routines are the function coxph() from the survival package (Therneau, 2017) and the function $\mathrm{cph}($ ) from the rms package (Harrell Jr, 2017). We present a fast and memory efficient algorithm to extract baseline hazards and predicted risks with confidence intervals from an object obtained with either of these functions.

In the presence of competing risks one needs to combine at least two Cox regression models to predict the absolute risk of an event (cumulative incidence) conditional on covariates (Benichou and Gail, 1990). We present the CSC()-function of the R package riskRegression which fits the Cox regression models using either $\operatorname{coxph}()$ or $\mathrm{cph}($ ). We also present a concomitant predict() S3 method which computes the absolute risks of the event of interest for given combinations of covariate values and time points. Optionally, the predict () method computes asymptotic confidence intervals and confidence bands for the predicted absolute risks. We review the formula behind the estimators implemented and illustrate the $\mathrm{R}$ interface.

It is possible to obtain the predictions of absolute risks based on cause-specific Cox regression also with the survival package or with the mstate package (Putter et al., 2016). However, both require more work from the user. Finally, it should be noted that there are alternative regression methods for absolute risks in the presence of competing risks such as Fine-Gray regression (Fine and Gray, 1999) or direct binomial regression (Gerds et al., 2012; Scheike et al., 2008).

\section{Data used for examples}

For the sole purpose of illustration we use the 'Melanoma' data set which is included in the riskRegression package. It contains data from 205 malignant melanoma patients. Among the risk factors for cancer specific death were patient age and sex and the histological variables tumor thickness, invasion (levels $0,1,2$ ), and epithelioid cells (no present vs. present). Within the limitation of the follow-up periods, it was observed that 57 patients had died from cancer ("status" equals 1) and 14 had died from other causes ("status" equals 2). The remaining patients were right censored ("status" equals 0 ).

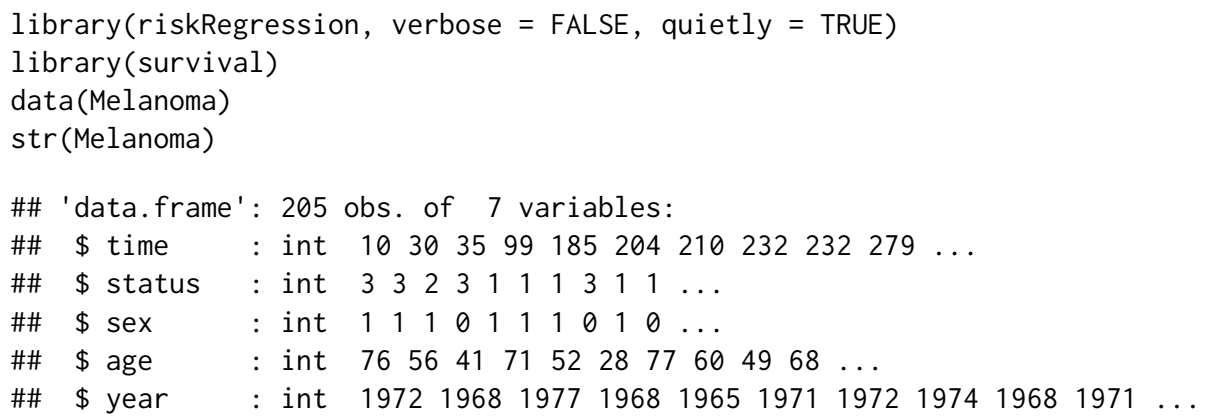




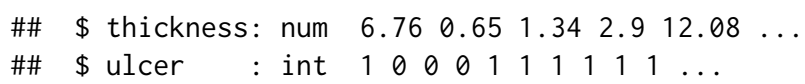

\section{Predicting absolute risks based on cause-specific Cox regression}

We denote by $T$ the time between a baseline date and the date of an event and by $D \in\{1, \ldots, K\}$ the cause of the event. We assume that $\{D=1\}$ is the event of interest. Let $X=\left(X^{1}, \ldots, X^{p}\right)$ be a $p$-dimensional vector of baseline covariates with arbitrary distribution, and $Z=\left(Z^{1}, \ldots, Z^{q}\right)$ be the strata variables, i.e. a set of categorical baseline covariates with finitely many possible values. Without loss of generality and to ease the notation we set $q=1$. We use $\{1, \ldots, L\}$ for the categories of $Z$.

We consider a setting in which the event time $T$ is right censored at a random time $C$. We assume that $C$ is conditionally independent of $T$ given $(X, Z)$ and fix a time $\tau$ such that almost surely $\mathrm{P}(C>\tau \mid X, Z)>0$. We denote $\tilde{T}=\min (T, C), \tilde{D}=\Delta D$, and $\Delta=1\{T \leq C\}$.

\section{Cause-specific Cox regression}

Given covariates $(X, Z)$, let $S_{0}(t \mid x, z)=P(T>t \mid X=x, Z=z)$ denote the event-free survival function and $F_{j}(t \mid x, z)=P(T \leq t, D=j \mid X=x, Z=z)$ the cumulative incidence function for event $j$. The cause-specific hazard rates are defined as $\lambda_{j, z}(t \mid x)=\mathrm{d} F_{j}(t \mid x, z) / S_{0}(t \mid x, z)$ (Andersen et al., 1993). We also denote the cumulative hazard rates by $\Lambda_{j, z}(t \mid x)=\int_{0}^{t} \lambda_{j, z}(s \mid x) \mathrm{d} s$. The stratified Cox regression model (Cox, 1972) for cause $j$ is given by

$$
\lambda_{j, z}(t \mid x)=\lambda_{0 j, z}(t) \exp \left(x \beta_{j}\right),
$$

where $\beta_{j}=\left(\beta_{j}^{1}, \ldots, \beta_{j}^{p}\right)^{\top}$ is a $p$-dimensional vector of regression coefficients (the log-hazard ratios), and $\left\{\lambda_{0 j, z}(t): z=1, \ldots, L\right\}$ a set of unspecified baseline hazard functions.

\section{Predicting the absolute risk of an event}

The cause-specific Cox regression models can be combined into a prediction of the absolute risk of an event of type 1 until time $t$ conditional on the covariates $x, z$. For the case where $K=2$ the absolute risk formula of Benichou and Gail (1990) is given by:

$$
F_{1}(t \mid x, z)=\int_{0}^{t} S(s-\mid x, z) \lambda_{1, z}(s \mid x) \mathrm{d} s
$$

where $s-$ denotes the right sided limit, e.g. $\Lambda_{1, z}(s-\mid x)=\lim _{v \rightarrow s, v<s} \Lambda_{1, z}(v \mid x)$. The absolute risk accumulates over time the product between the event-free survival and the hazard of experiencing the event of interest, both conditional to the baseline covariates and to the strata variable. The event free survival can be estimated from the cause-specific hazards using the product integral estimator:

$$
S(t \mid x, z)=\prod_{s \leq t}\left(1-d \Lambda_{1, z}(t \mid x)-d \Lambda_{2, z}(t \mid x)\right)
$$

or the exponential approximation:

$$
\widehat{S}(t \mid x, z)=\exp \left[-\widehat{\Lambda}_{1, z}(t \mid x)-\widehat{\Lambda}_{2, z}(t \mid x)\right]
$$

which is asymptotically equivalent to the product-limit estimator if the distribution of the event times is continuous. Using the product integral estimator ensures that $S(t \mid x, z)+F_{1}(t \mid x, z)+F_{2}(t \mid x, z)$ equals exactly 1 . This is a desirable property since the sum of the transition probabilities over all possible transitions should sum to one.

Formula (2) generalizes to situations with more than 2 competing risks, i.e., $K>2$. However, in applications with many competing risks there will sometimes be few events of specific causes, and it may be hard to fit a Cox regression model for each cause separately. One possibility when $K>2$ is to combine all causes where $\tilde{D}>1$ into a single competing risk for the cause of interest $\tilde{D}=1$. While the riskRegression package allows the use of more than 2 competing risks, we will illustrate its use considering only 2 competing risks. The package implements formula (2) in two steps. 


\section{Step 1: estimation of the cause-specific hazards}

The first step is to fit the Cox regression models with the $\operatorname{CSC}()$ function in order to estimate $\lambda_{1, z}$ and $\lambda_{2, z}$ :

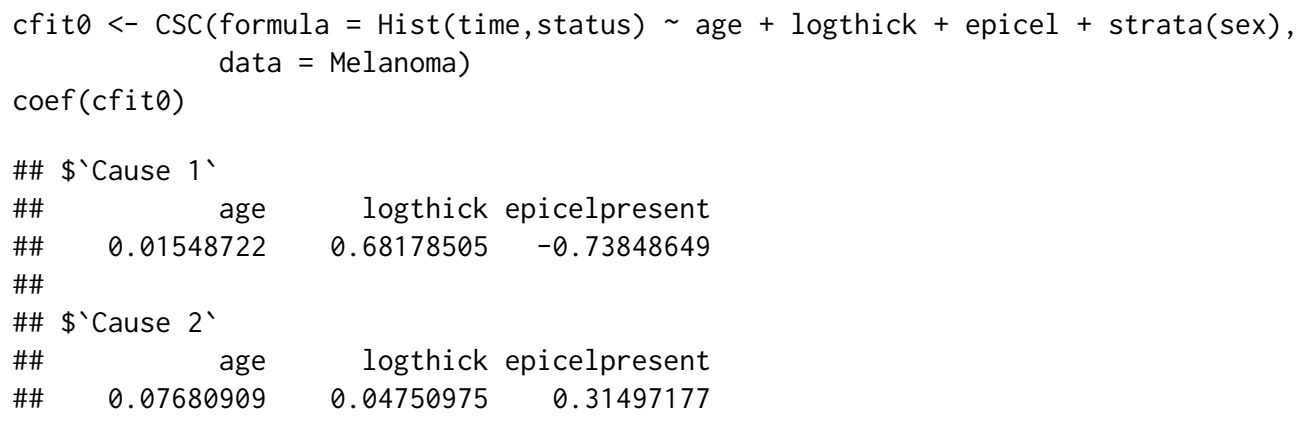

In the call of $\csc ()$ the argument formula is used to define the outcome variables with the help of the function prodlim: : Hist(). The variable "time" in the data set contains the values of the observed event time $\tilde{T}$ and the variable "status" the cause of the event $\tilde{D}$. Objects generated with the function prodlim: :Hist() have a print method:

h <- with(Melanoma, prodlim: :Hist(time, status))

$\mathrm{h}$

\#\# Right-censored response of a competing.risks model

\#\#

\#\# No.Observations: 205

\#\#

\#\# Pattern:

\#\#

\#\# Cause event right.censored

\#\# $1157 \quad 0$

$\begin{array}{llll}\# \# & 2 & 14 & 0\end{array}$

\# unknown 0

and a plot method:

plot $(\mathrm{h}$, arrowLabelstyle $=$ "count", stateLabels = c("Radical \noperation", "Cancer $\backslash$ nrelated death", "Death $\backslash$ nother causes"))

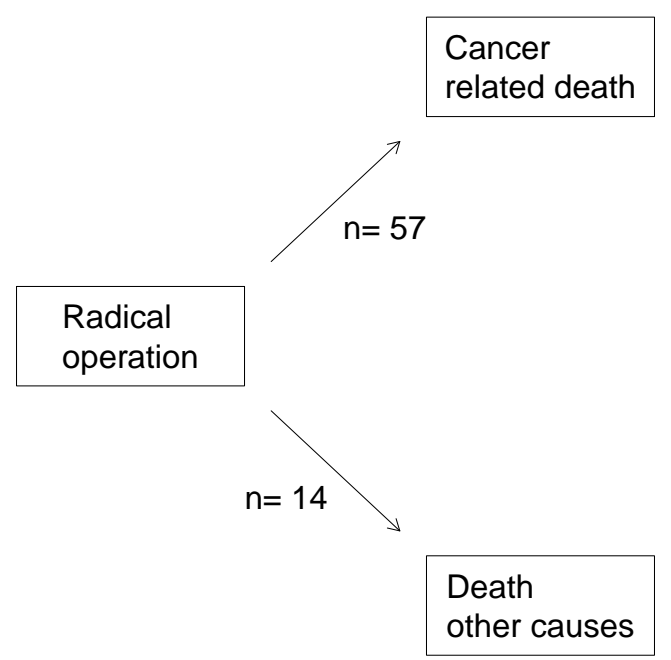

Figure 1: Box-arrow diagram showing the three states of the competing risk model and the number of observed transitions in the Melanoma data set.

A nice complement to the regression models is the marginal Aalen-Johansen estimate of the absolute risk of cancer related death (Figure 2): 

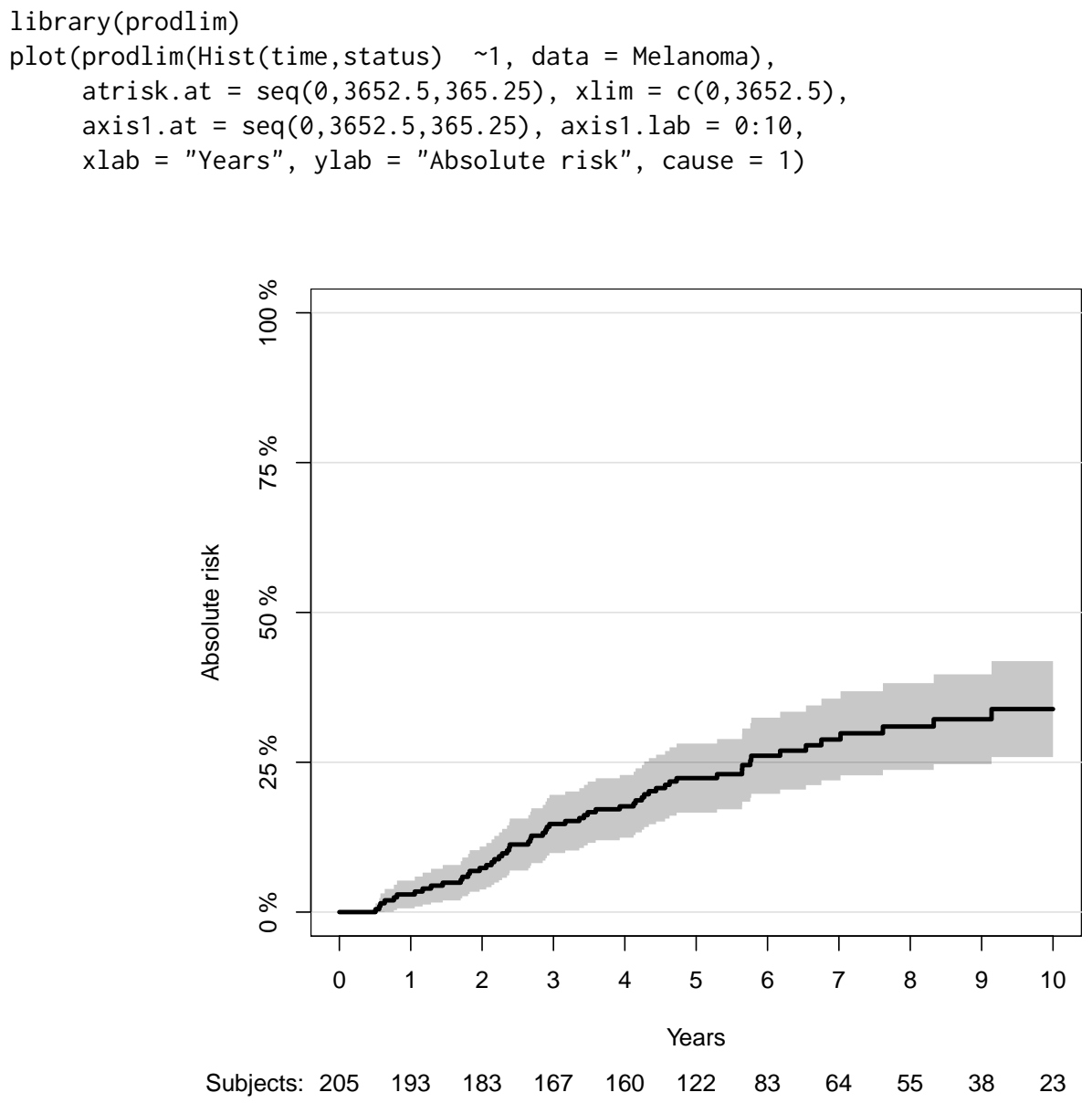

Figure 2: Non-parametric estimation of the absolute risk of cancer related death over time obtained using the Aalen-Johansen estimator.

The right hand side of the formula in the call of the $\csc ()$ function:

Hist(time,status) age + logthick + epicel + strata(sex)

defines the covariate(s) $X$ which enter into the linear predictor $x \beta$ in formula (1), and the strata variable(s) $Z$ which define the baseline hazard functions $\lambda_{0 j, z}$. Strata variables are specified by wrapping the variable names into the special function strata(), as one would do when using the $\operatorname{coxph}()$ function. If only one formula is provided, the $\csc ()$ function will use the same baseline covariates and strata variables for all cause-specific Cox regression models. Instead one may feed a list of formulas into the argument formula, one for each cause:

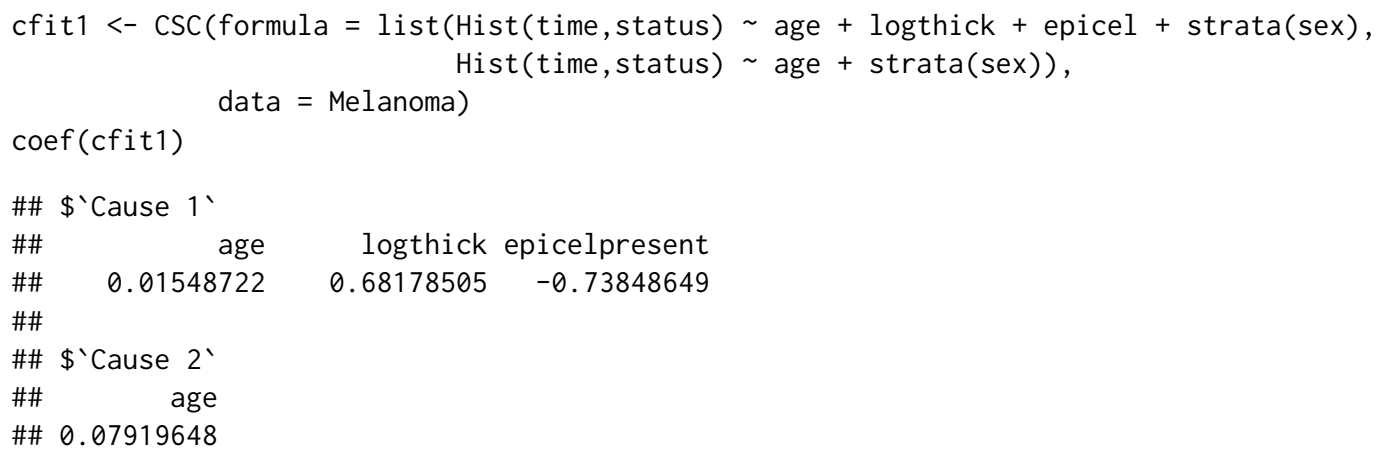

Note that the choice of the baseline covariates relative to each cause made here is not based on clinical or statistical criteria; it was done to illustrate the software possibilities. The causes are internally ordered with respect to the levels of the variable "status", if this variable is a factor, and otherwise with respect to sort (as. character (unique(status))). The order of the causes is saved as cfit1[["causes"]]. Accordingly, the first formula is used to fit a Cox regression model to the first cause and the second formula is used to fit a Cox regression model to the second cause and so on. 
Internally, $\csc ()$ constructs dummy variables, one for each cause, and then calls the function defined by the argument fitter on a suitably constructed Surv() formula. By default the cause-specific Cox models are fitted with the function survival: $: \operatorname{coxph}()$. Alternatively, one can set the argument fitter to the name of a different routine, e.g., cph.

\section{Step 2: computation of the absolute risk}

The object obtained with $\csc ()$ has class "CauseSpecificCox". The second step is to call the corresponding predict () method. In addition to the object obtained with $\csc ()$ this requires three additional arguments: newdata, times, cause. The argument newdata should be a "data.frame" which contains the covariates $X$ and $Z$ in the same format as the data used to fit $\operatorname{CSC}()$. The argument cause defines the cause of interest $D$ and the argument times defines a vector of prediction horizon(s) whose values are used as the upper integration limit $t$ in formula (2). The predict () method computes the absolute risks (formula (2)) for each row in newdata and each value of times:

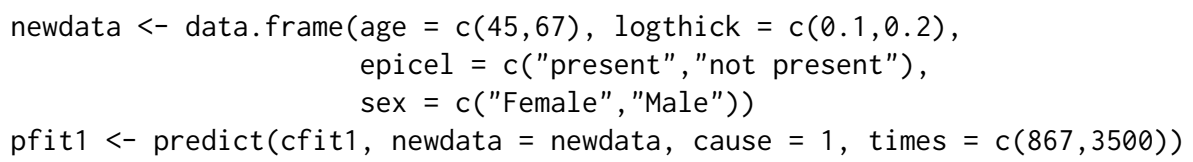

By default, the product integral estimator is used to estimate the event-free survival function. Setting the argument productLimit to FALSE when calling the predict function enables to use the exponential approximation. The predict function returns a structured list of class "predictCSC". The corresponding print () method calls as.data.table.predictcsc() to display the predictions as follows:

print(pfit1)

$\begin{array}{lrrrrrrrr}\text { \#\# } & \text { observation } & \text { age } & \text { logthick } & \text { epicel } & \text { sex times } & \text { strata } & \text { absRisk } \\ \text { \#\# 1: } & 1 & 45 & 0.1 & \text { present } & \text { Female } & 867 & \text { sex=Female } & 0.021 \\ \text { \#\# 2: } & 2 & 67 & 0.2 \text { not present } \text { Male } & 867 & \text { sex=Male } & 0.149 \\ \text { \#\# 3: } & 1 & 45 & 0.1 & \text { present } & \text { Female } & 3500 & \text { sex=Female } & 0.117 \\ \text { \#\# 4: } & 2 & 67 & 0.2 \text { not present } \text { Male } & 3500 & \text { sex=Male } & 0.428\end{array}$

For each row in newdata (values are repeated for each prediction horizon) and each prediction horizon (column times) the column "absRisk" contains the absolute risk of cancer specific mortality (cause 1). Standard errors and confidence intervals for the absolute risk can be obtained setting the argument se to TRUE:

\begin{tabular}{|c|c|c|c|c|c|c|c|}
\hline \#\# & observation & times & strata & absRisk & absRisk.se & absRisk. lower & absRisk.upper \\
\hline \#\# 1: & 1 & 867 & sex $=$ Female & 0.021 & 0.122 & 0.00738 & 0.0478 \\
\hline \#\# 2: & 2 & 867 & sex $=$ Male & 0.149 & 0.161 & 0.07356 & 0.2502 \\
\hline \#\# 3: & 1 & 3500 & sex $=$ Female & 0.117 & 0.151 & 0.05552 & 0.2025 \\
\hline \#\# 4: & 2 & 3500 & sex=Male & 0.428 & 0.320 & 0.20416 & 0.6355 \\
\hline
\end{tabular}

Here we have set the argument keep. newdata to FALSE to not export the value of the covariates. The structure of the "predictCSC" object is as follows.

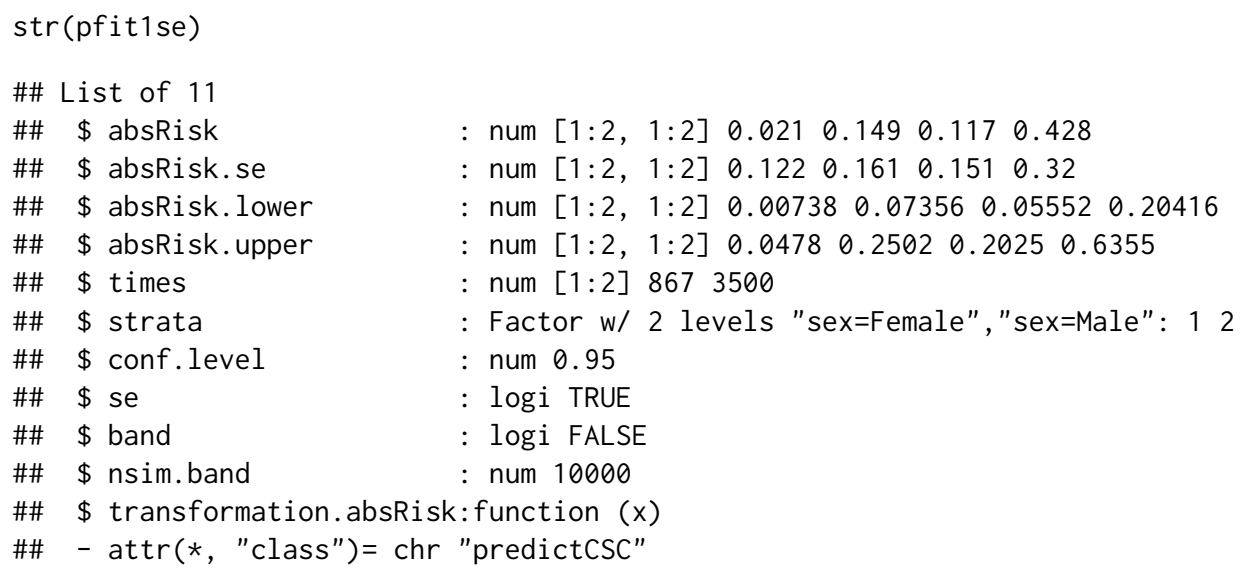


The elements \$absRisk, \$absRisk. se, \$absRisk. lower and \$absRisk. upper are matrices where each row corresponds to a row in newdata and each column to a value of the times vector. All these matrices are sorted according to the original orders of the arguments newdata and times. To conveniently extract a subset of the results, one should first call as.data.table.predictcsc() to combine these results into a "data. table" object. Here is an example:

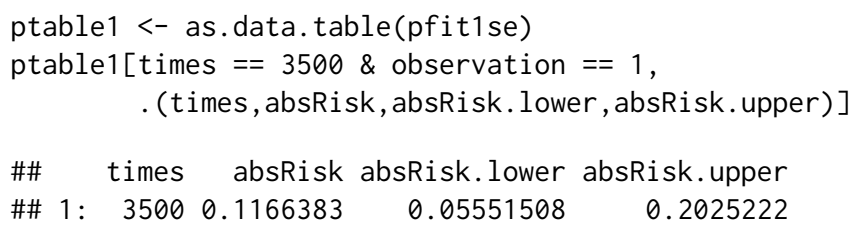

In the same way confidence bands can be obtained by setting the argument band to TRUE:

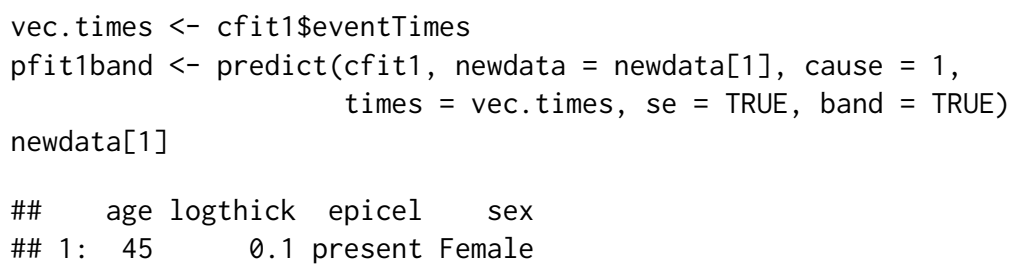

By default 10,000 simulations will be used to estimate the appropriate quantile for the confidence bands (see explanations in section Construction of the confidence bands). This default behavior can be changed by setting the argument nsim. band to another value. The autoplot() function can then be used to compare confidence bands and the confidence intervals:
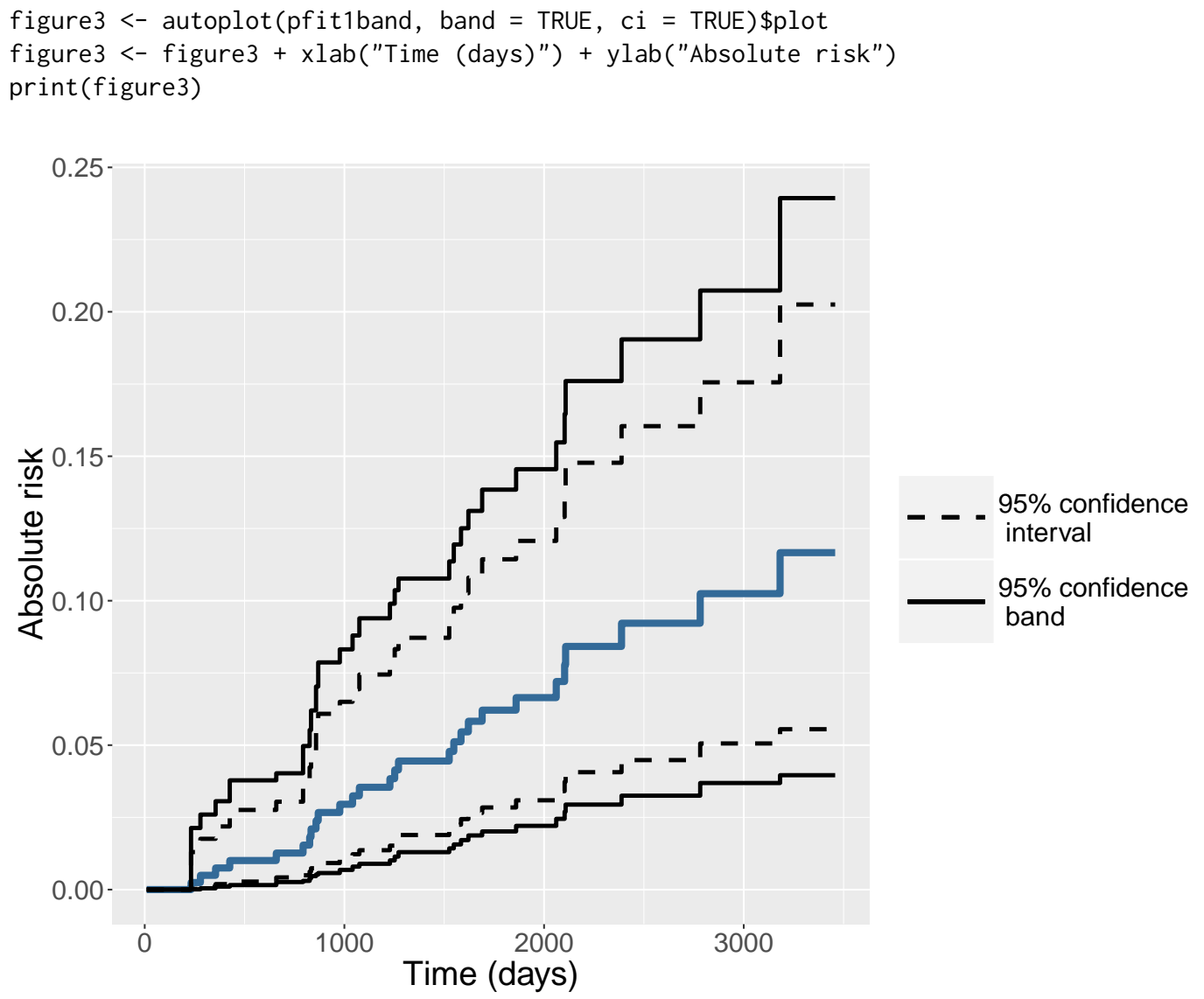

Figure 3: Absolute risk over time for a 45 years old female patient with a tumor thickness of $0.1 \mathrm{~mm}$ and epithelioid cells (blue line). The continuous black lines represent the confidence bands while the dashed black lines represent the range of the confidence intervals.

Note that the resulting object is a "ggplot" graphic. This can be useful to personalize the graph, e.g. change the font size of the text. 


\section{Construction of the confidence intervals}

In this section we describe the asymptotic formula behind the confidence intervals for the predicted absolute risks and the empirical counterpart which is implemented in riskRegression. We assume a sample $\left(\mathcal{X}_{i}\right)_{i \in\{1, \ldots, n\}}$ of $n$ independent and identically distributed replications of $\mathcal{X}=(\tilde{T}, \tilde{D}, X, Z)$. The estimator $\hat{F}_{1}$ of $F_{1}$ is obtained by substituting the Cox partial likelihood estimate $\hat{\beta}_{j}$ for $\beta_{j}$ and the baseline hazard estimate $\hat{\lambda}_{0 j, z}$ for $\lambda_{0 j, z}$ in equations (1) and (2).

The asymptotic confidence intervals for the covariate specific absolute risks of event 1 before time $t$ are based on the following von Mises expansion (van der Vaart, 1998):

$$
\sqrt{n}\left(\hat{F}_{1}(t \mid x, z)-F_{1}(t \mid x, z)\right)=\frac{1}{\sqrt{n}} \sum_{i=1}^{n} \phi_{F_{1}}\left(\mathcal{X}_{i} ; t, x, z\right)+o_{\mathrm{P}}(1) .
$$

where the exponential approximation is used for defining the event free survival in $F_{1}(t \mid x, z)$. Given (4), for fixed values $t, x, z$ the central limit theorem implies that $\hat{F}_{1}(t \mid x, z)$ has an asymptotic normal distribution with asymptotic variance $V_{F_{1}}(t, x, z)=\mathrm{E}\left(\phi_{F_{1}}\left(\mathcal{X}_{i} ; t, x, z\right)^{2}\right)$. Based on the estimate $\widehat{\phi}_{F_{1}}$ of the influence function $\phi_{F_{1}}$ (both defined in subsequent subsections) our variance estimate is given by

$$
\widehat{V}(t, x, z)=\frac{1}{n} \sum_{i=1}^{n} \widehat{\phi}_{F_{1}}\left(\mathcal{X}_{i} ; t, x, z\right)^{2} .
$$

We then construct Wald confidence intervals for $F_{1}(t \mid x, z)$ in the usual way:

$$
\left[F_{1}(t \mid x, z)+q_{\alpha / 2} \sqrt{\widehat{V}(t, x, z))} ; F_{1}(t \mid x, z)+q_{1-\alpha / 2} \sqrt{\widehat{V}(t, x, z)}\right]
$$

where $q_{\alpha}$ is the $\alpha$-quantile of the normal distribution. Since the absolute risk is bounded below by 0 and above by 1 , the confidence interval is automatically restricted to this interval. Alternatively the confidence interval can be computed using a log-log transformation: first the confidence interval is computed on the log-log scale:

$$
\left[\log \left(-\log \left(F_{1}(t \mid x, z)\right)\right)+q_{\alpha / 2} \sqrt{\left.\widehat{V}_{\log -\log }(t, x, z)\right)} ; \log \left(-\log \left(F_{1}(t \mid x, z)\right)\right)-q_{1-\alpha / 2} \sqrt{\widehat{V}_{\log -\log }(t, x, z)}\right]
$$

where $V_{\log -\log }$ is the variance of the influence function on the log-log scale. Then the confidence interval is back transformed using the link function: $x \mapsto \exp (-\exp (x))$. This ensures that the confidence

\begin{tabular}{|c|c|c|c|c|c|c|c|}
\hline \#\# & observation & times & strata & absRisk & absRisk.se & absRisk.lower & absRisk.upper \\
\hline \#\# 1: & 1 & 867 & sex=Female & 0.021 & 0.00992 & 0.00157 & 0.0404 \\
\hline \#\# 2: & 2 & 867 & sex $=$ Male & 0.149 & 0.04586 & 0.05945 & 0.2392 \\
\hline \#\# 3: & 1 & 3500 & sex=Female & 0.117 & 0.03795 & 0.04226 & 0.1910 \\
\hline \#\# 4: & 2 & 3500 & sex=Male & 0.428 & 0.11620 & 0.20021 & 0.6557 \\
\hline
\end{tabular}
interval is bounded below by 0 and above by 1 . By default, the confidence intervals are computed using the log-log transformation. To compute them without using the log-log transformation, the argument log. transform needs to be set to FALSE when calling predict. CauseSpecificCox():

Here the column "absRisk.se" contains $V_{\log -\log }$ (log-log scale) while the columns "absRisk", "absRisk.lower", and "absRisk.upper" are on the original scale. The benefit of using a log-log transformation is studied in the section Coverage of the confidence interval and the confidence bands.

\section{Asymptotic formula}

The influence function $\phi_{F_{1}}$ can be expressed as a function of the influence functions $\phi_{\lambda_{1}}$ and $\phi_{\lambda_{2}}$ of the cause-specific hazard rates:

$$
\begin{aligned}
\phi_{F_{1}}(\mathcal{X}, t, x, z)= & \int_{0}^{t} \exp \left(-\Lambda_{1, z}(s \mid x)-\Lambda_{2, z}(s \mid x)\right) d \phi_{\Lambda_{1, z}}(\mathcal{X} ; s, x) \\
& -\int_{0}^{t} \lambda_{1, z}(s \mid x) \exp \left(-\Lambda_{1, z}(s \mid x)-\Lambda_{2, z}(s \mid x)\right)\left(\phi_{\Lambda_{1, z}}(\mathcal{X} ; s, x)+\phi_{\Lambda_{2, z}}(\mathcal{X} ; s, x)\right) d s .
\end{aligned}
$$


We use the shorthand notations $v^{\otimes 0}=0, v^{\otimes 1}=v, v^{\otimes 2}=v v^{\top}$ and

$$
\int f(s, d, v, w) \mathrm{dP}(s, d, v, w)=\int_{0}^{\infty} \sum_{d=0}^{K} \int_{R^{p}} \sum_{z=1}^{L} f(s, d, v, w) \mathrm{dP}(s, d, v, w) .
$$

We also suppress the dependence on $\tau$ when we in the following adapt the usual notation for Cox model asymptotic theory to the cause-specific and stratified case:

$$
\begin{aligned}
\mathcal{S}^{(r)}\left(t, \beta_{j}, z\right) & =\int 1\{t \leq s \leq \tau, w=z\} \exp \left(v \beta_{j}\right) v^{\otimes r} \mathrm{dP}(s, d, v, w) \\
E\left(t, \beta_{j}, z\right) & =\frac{\mathcal{S}^{(1)}\left(t, \beta_{j}, z\right)}{\mathcal{S}^{(0)}\left(t, \beta_{j}, z\right)} \\
\mathcal{I}\left(\beta_{j}\right) & =\int 1\{d=j\}\left(\frac{\mathcal{S}^{(2)}\left(s, \beta_{l}, w\right)}{\mathcal{S}^{(0)}\left(s, \beta_{j}, w\right)}-E\left(s, \beta_{j}, w\right)^{\otimes 2}\right) \mathrm{dP}(s, d, v, w) .
\end{aligned}
$$

For fixed cause $j$, time $t$ and strata $z$ the expansions $\sqrt{n}\left(\hat{\beta}_{j}-\beta_{j}\right)=\frac{1}{\sqrt{n}} \sum_{i=1}^{n} \phi_{\beta_{j}}\left(\mathcal{X}_{i}\right)+o_{\mathrm{P}}(1)$ and $\sqrt{n}\left(\hat{\Lambda}_{0 j, z}(t)-\Lambda_{0 j, z}(t)\right)=\frac{1}{\sqrt{n}} \sum_{i=1}^{n} \phi_{\Lambda_{0 j, z}}\left(\mathcal{X}_{i}, t\right)+o_{\mathrm{P}}(1)$ are then characterized by the influence functions

$$
\begin{aligned}
\phi_{\beta_{j}}(\mathcal{X})= & \mathcal{I}\left(\beta_{j}\right)^{-1}\left(1\{\tilde{D}=j, \tilde{T}<\tau\}\left(X-E\left(\tilde{T}, \beta_{j}, Z\right)\right)\right. \\
& \left.-\exp \left(X \beta_{j}\right) \int 1\{d=j, s \leq \tilde{T}\} \frac{X-E\left(s, \beta_{j}, Z\right)}{\mathcal{S}^{(0)}\left(s, \beta_{j}, Z\right)} \mathrm{d} P(s, d, v, w)\right) \\
\phi_{\Lambda_{0 j, z}}(\mathcal{X} ; t)= & -\phi_{\beta_{j}}(\mathcal{X}) \int_{0}^{t} E\left(s, \beta_{j}, z\right) \lambda_{0 j, z}(s) d s \\
& -1\{Z=z\}\left(\exp \left(X \beta_{j}\right) \int_{0}^{\min (t, \tilde{T})} \frac{\lambda_{0 j, z}(s)}{\mathcal{S}^{(0)}\left(s, \beta_{j}, z\right)} d s-\frac{1\{\tilde{T} \leq t, \tilde{D}=j\}}{\mathcal{S}^{(0)}\left(\tilde{T}, \beta_{j}, Z\right)}\right) .
\end{aligned}
$$

In absence of strata, formula (7) is equal to formula 2 of (Reid, 1981) and formula (8) equals the one given in Gerds and Schumacher (2001, top of page 576; note however that there is a sign mistake in their first term). To connect these formulas with formula (6) it remains to note that under the Cox regression model the influence function of the cause-specific hazard rate can be written as:

$$
\phi_{\Lambda_{j, z}}(\mathcal{X} ; t, x)=\exp \left(x \beta_{j}\right)\left(\phi_{\Lambda_{0 j, z}}(\mathcal{X} ; t)+\Lambda_{0 j, z}(t) x \phi_{\beta_{j}}(\mathcal{X})^{\top}\right) .
$$

\section{Empirical estimates}

The following formulas are obtained with the plug-in principle substituting the Cox partial likelihood estimates $\hat{\beta}_{j}$ for $\beta_{j}$ and the baseline hazard estimates $\hat{\lambda}_{0 j, z}$ for $\lambda_{0 j, z}$ into formulas (6) - (9). We denote by $N_{j}^{z}(t)=\sum_{i=1}^{n} 1\left\{\tilde{T}_{i} \leq t, \tilde{D}_{i}=j, Z_{i}=z\right\}$ the strata and cause specific counting process, and by $Y^{z}(t)=\sum_{i=1}^{n} 1\left\{T_{i} \geq t, Z_{i}=z\right\}$ the strata specific "at-risk" process (Andersen et al., 1993). The empirical estimate of the influence function of the partial likelihood estimate is given by

$$
\widehat{\phi}_{\beta_{j}}\left(\mathcal{X}_{i}\right)=\widehat{\mathcal{I}}\left(\beta_{j}\right)^{-1}\left(\Delta_{i}\left(X_{i}-\hat{E}\left(\tilde{T}_{i}, \hat{\beta}_{j}, Z_{i}\right)\right)-\exp \left(X_{i} \hat{\beta}_{j}\right) \int_{0}^{T_{i}} \frac{X_{i}-\hat{E}\left(s, \hat{\beta}_{j}, Z_{i}\right)}{\widehat{\mathcal{S}^{(0)}}\left(s, \hat{\beta}_{j}, Z_{i}\right)} \mathrm{d} N_{j}(s)\right)
$$

where

$$
\widehat{\mathcal{S}^{(r)}}\left(t, \hat{\beta}_{j}, z\right)=\int_{0}^{\tau} \exp \left(X_{i} \hat{\beta}_{j}\right) X_{i}^{\otimes r} \mathrm{~d} N_{j}^{z}(t)
$$


The influence functions for the cumulative baseline hazard and its first differential are estimated by:

$$
\begin{aligned}
\widehat{\phi}_{\Lambda_{0 j, z}}\left(\mathcal{X}_{i} ; t\right)= & -\widehat{\phi}_{\beta_{j}}\left(\mathcal{X}_{i}\right) \int_{0}^{t} E\left(s, \hat{\beta}_{j}, z\right) \hat{\lambda}_{0 j, z}(s) \mathrm{d} s \\
& -1\left\{Z_{i}=z\right\}\left(\exp \left(X_{i} \hat{\beta}_{j}\right) \int_{0}^{\min \left(t, \tilde{T}_{i}\right)} \frac{\hat{\lambda}_{0 j, z}(s)}{\widehat{\mathcal{S}^{(0)}}\left(s, \hat{\beta}_{j}, z\right)} d s-\frac{1\left\{\tilde{T}_{i} \leq t, \tilde{D}_{i}=j\right\}}{\widehat{\mathcal{S}^{(0)}}\left(\tilde{T}_{i}, \hat{\beta}_{j}, Z_{i}\right)}\right) \\
d \widehat{\phi}_{\Lambda_{0 j, z}}\left(\mathcal{X}_{i} ; t\right)= & -\widehat{\phi}_{\beta_{j}}\left(\mathcal{X}_{i}\right) E\left(t, \hat{\beta}_{j}, z\right) \hat{\lambda}_{0 j, z}(t) \\
& -1\left\{Z_{i}=z\right\}\left(\exp \left(X_{i} \hat{\beta}_{j}\right) 1\left\{t \leq \tilde{T}_{i}\right\} \frac{\hat{\lambda}_{0 j, z}(t)}{\widehat{\mathcal{S}^{(0)}}\left(t, \hat{\beta}_{j}, z\right)}-\frac{1\left\{\tilde{T}_{i}=t, \tilde{D}_{i}=j\right\}}{\widehat{\mathcal{S}^{(0)}}\left(\tilde{T}_{i}, \hat{\beta}_{j}, Z_{i}\right)}\right) .
\end{aligned}
$$

These estimates lead to the following estimates of the influence functions of the covariate specific cumulative hazard and its derivative relative to the time:

$$
\begin{aligned}
\hat{\phi}_{\Lambda_{j, z}}\left(\mathcal{X}_{i} ; t, x\right) & =\exp \left(x \hat{\beta}_{j}\right)\left(\widehat{\phi}_{\Lambda_{0 j, z}}\left(\mathcal{X}_{i} ; t\right)+\hat{\Lambda}_{0 j, z}(t) x \widehat{\phi}_{\beta_{j}}\left(\mathcal{X}_{i}\right)^{\top}\right) \\
d \hat{\phi}_{\Lambda_{j, z}}\left(\mathcal{X}_{i} ; t, x\right) & =\exp \left(x \hat{\beta}_{j}\right)\left(d \widehat{\phi}_{\Lambda_{0 j, z}}\left(\mathcal{X}_{i} ; t\right)+\hat{\lambda}_{0 j, z}(t) x \widehat{\phi}_{\beta_{j}}\left(\mathcal{X}_{i}\right)^{\top}\right) .
\end{aligned}
$$

Finally, we obtain our estimate of the influence function of the absolute risk:

$$
\begin{aligned}
& \widehat{\phi}_{F_{1}}\left(\mathcal{X}_{i}, t, x, z\right)=\int_{0}^{t} \exp \left(-\hat{\Lambda}_{1, z}(s \mid x)-\hat{\Lambda}_{2, z}(s \mid x)\right) d \widehat{\phi}_{\Lambda_{1, z}}\left(\mathcal{X}_{i} ; s, x\right) \\
& -\int_{0}^{t} \hat{\lambda}_{1, z}(s \mid x) \exp \left(-\hat{\Lambda}_{1, z}(s \mid x)-\hat{\Lambda}_{2, z}(s \mid x)\right)\left(\widehat{\phi}_{\Lambda_{1, z}}\left(\mathcal{X}_{i} ; s, x\right)+\widehat{\phi}_{\Lambda_{2, z}}\left(\mathcal{X}_{i} ; s, x\right)\right) \mathrm{d} s .
\end{aligned}
$$

\section{Construction of the confidence bands}

A confidence band with confidence level $1-\alpha$ for the absolute risk $F_{1}(. \mid x, z)$ restricted to the time interval $\mathcal{T}=\left[\tau_{1} ; \tau_{2}\right]$ is a region $R_{\mathcal{T}}(x, z)=\left[l_{x, z}(t) ; u_{x, z}(t)\right]_{t \in \mathcal{T}}$ satisfying:

$$
\mathrm{P}\left(F_{1}(t \mid x, z) \in\left[l_{x, z}(t) ; u_{x, z}(t)\right] \mid \forall t \in \mathcal{T}\right)=1-\alpha .
$$

In figure Figure $3, \tau_{1}=0$ and $\tau_{2}=3458$, the time at which the last event occurred. Using the martingale central limit theorem, (Cheng et al., 1998) have shown that $\hat{F}_{1}(t \mid x, z)-F_{1}(t \mid x, z)$ converges weakly to a zero-mean Gaussian process on $\mathcal{T}$. The asymptotic variance of this process is $V(t, x, z)$. However the $1-\alpha$ quantile achieving simultaneous coverage is larger than the $1-\alpha$ quantile of a standard normal distribution. Since the dependence between the increments of the process $\hat{F}_{1}(t \mid x, z)-$ $F_{1}(t \mid x, z)$ makes the derivation of an explicit expression for the quantile difficult, we used instead a resampling technique (Scheike and Zhang, 2008). Consider over $t \in \mathcal{T}$ the normalized process:

$$
\psi_{F_{1}}\left(\mathcal{X}_{i} ; t, x, z\right)=\phi_{F_{1}}\left(\mathcal{X}_{i} ; t, x, z\right) / \sqrt{V(t, x, z)} .
$$

Denote by $c_{1-\alpha / 2}$ the $1-\alpha / 2$ quantile of the sample:

$$
\sup _{t \in \mathcal{T}}\left|\psi_{F_{1}}\left(\mathcal{X}_{i} ; t, x, z\right)\right|
$$

and using the symmetry of the Gaussian distribution, i.e. $c_{\alpha / 2}=-c_{1-\alpha / 2}$, a $1-\alpha$ confidence band over $\mathcal{T}$ is constructed as follows:

$$
\left[F_{1}(t \mid x, z)-c_{1-\alpha / 2} \sqrt{\widehat{V}(t, x, z))} ; F_{1}(t \mid x, z)+c_{1-\alpha / 2} \sqrt{\widehat{V}(t, x, z)}\right] .
$$

Like for the confidence intervals, the confidence bands will be restricted to the interval [0;1] when they are not computed using a log-log transformation.

\section{Implementation details}

The function predict. CauseSpecificCox() calls two important functions, predictCox() that computes the hazard and cumulative hazard for a fitted Cox model, and iidCox() that computes the influence function for the baseline hazard and regression coefficients. In this section we first explain 
how predictCox () deals with ties in the event times. We then show that the function predictCox() can also be used to obtain confidence intervals and bands for covariate specific survival probabilities in the situation without competing risks. Implementation details about the function iidCox() are postponed to appendix B. These details may be useful for programmers who need to care about memory usage.

\section{Handling of tied event times}

We speak of ties when two or more observations have the same value of the time variable $\tilde{T}$. Ties occur for example when time is recorded on a discrete scale such as months. The survival package implements three different methods to deal with ties ("efron", "breslow", and "exact", see help(coxph)) for the partial likelihood estimator of the $\log$ hazard ratios $\beta_{j}$ (Therneau and Grambsch, 2000). We have implemented the "efron" and the "breslow" method but not the "exact" method. For a comparison of these methods and yet another method see Scheike and Sun (2007). We now state the formula for the baseline hazard function under Breslow's (Breslow, 1974) and Efron's method (Efron, 1977) for the handling of ties. The baseline hazard estimate in strata $z$ given by the Breslow method is:

$$
d \hat{\Lambda}_{0 j, z}^{B}(t)=\frac{\mathrm{d} N_{j}^{z}(t)}{\sum_{i \in Y^{z}(t)} \exp \left(\beta^{t} X_{i}\right)} .
$$

With the Efron method for handling ties the formula is given by:

$$
d \hat{\Lambda}_{0 j, z}^{E}(t)=\sum_{k=1}^{\mathrm{d} N_{j}^{z}(t)} \frac{1}{\sum_{i \in Y^{z}(t)} \exp \left(\beta^{t} X_{i}\right)-\frac{k-1}{\mathrm{~d} N_{j}^{z}(t)} \sum_{i=1}^{\mathrm{d} N_{j}^{z}(t)} \exp \left(\beta^{t} X_{i}\right)} .
$$

Both estimators are implemented in the function predictCox() which provides estimates of the baseline hazard, the cumulative baseline hazard and baseline survival. The predictCox() function does not have an argument to specify whether Breslow method or Efron method should be used; instead it uses the same method that has been used to estimate the regression coefficients. In the case of the $\operatorname{coxph}()$ function, the default method is Efron:

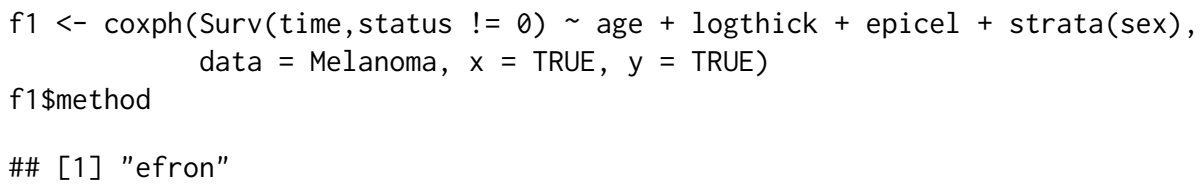

Therefore the baseline hazard will be estimated using the Efron method when calling predictCox():

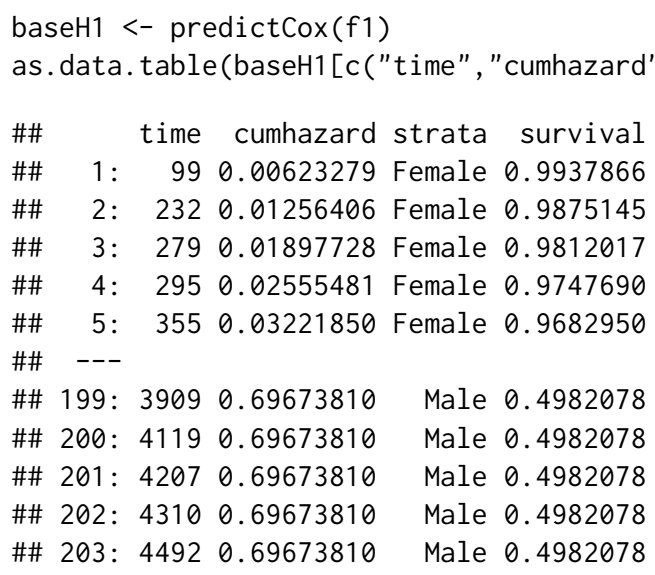

The cumulative baseline hazard and baseline survival are displayed in the columns "cumHazard", and "survival" of the output. This corresponds, respectively, to $\Lambda_{0 j, z}(t)$ and $\exp \left(-\Lambda_{0 j, z}(t)\right)$ where $j$ is $1, z$ can be found in the column "strata" and $t$ in "time". The covariate specific cumulative hazard $\Lambda_{j, z}(t \mid x)$ and survival $\exp \left(-\Lambda_{j, z}(t \mid x)\right)$ can be estimated using the same function:

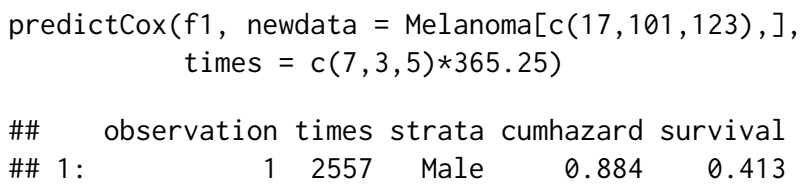




$\begin{array}{llrrrr}\text { \#\# 2: } & 2 & 2557 & \text { Female } & 0.555 & 0.574 \\ \text { \#\# 3: } & 3 & 2557 & \text { Female } & 0.949 & 0.387 \\ \text { \#\# 4: } & 1 & 1096 & \text { Male } & 0.453 & 0.635 \\ \text { \#\# 5: } & 2 & 1096 \text { Female } & 0.202 & 0.817 \\ \text { \#\# 6: } & 3 & 1096 & \text { Female } & 0.346 & 0.708 \\ \text { \#\# 7: } & 1 & 1826 \text { Male } & 0.670 & 0.512 \\ \text { \#\# 8: } & 2 & 1826 \text { Female } & 0.366 & 0.693 \\ \text { \#\# 9: } & 3 & 1826 \text { Female } & 0.626 & 0.535\end{array}$

\section{Confidence intervals and confidence bands for survival probabilities}

In absence of competing risks, the influence function of a Cox model can be used to estimate confidence intervals for the survival. This can be done by setting the argument se to TRUE when calling the predictCox() function:

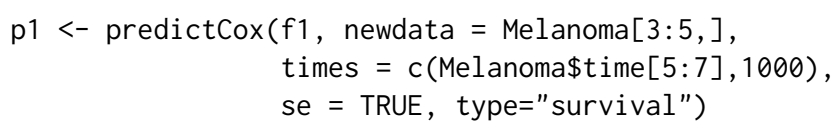

The predictCox() function output an object of class "predictCox". The print() method can be used to display the confidence intervals for the survival computed at different times:

$\operatorname{print}(\mathrm{p} 1)$

\begin{tabular}{|c|c|c|c|c|c|c|c|c|}
\hline \#\# & & observation & times & sti & urvival & surviva & surv? & surv \\
\hline \#\# & 1: & 1 & 185 & Male & 0.980 & 0.616 & 0.935 & 0.994 \\
\hline \#\# & 2: & 2 & 185 & Female & 0.985 & 1.014 & 0.893 & 0.998 \\
\hline \#\# & 3: & 3 & 185 & Male & 0.947 & 0.652 & 0.823 & 0.985 \\
\hline \#\# & 4: & 1 & 204 & Male & 0.973 & 0.567 & 0.921 & 0.991 \\
\hline \#\# & 5: & 2 & 204 & Female & 0.985 & 1.014 & 0.893 & 0.998 \\
\hline \#\# & 6: & 3 & 204 & Male & 0.929 & 0.594 & 0.791 & 0.977 \\
\hline \#\# & 7: & 1 & 210 & Male & 0.967 & 0.503 & 0.913 & 0.987 \\
\hline \#\# & 8: & 2 & 210 & Female & 0.985 & 1.014 & 0.893 & 0.998 \\
\hline \#\# & 9: & 3 & 210 & Male & 0.912 & 0.552 & 0.762 & 0.969 \\
\hline \#\# & 10: & 1 & 1000 & Male & 0.864 & 0.321 & 0.760 & 0.925 \\
\hline \#\# & 11: & 2 & 1000 & Female & 0.769 & 0.286 & 0.631 & 0.860 \\
\hline \#\# & 12: & 3 & 1000 & Male & 0.673 & 0.391 & 0.426 & 0.832 \\
\hline
\end{tabular}

Here the confidence intervals were computed using a log-log transformation. The argument log. transform can be set to FALSE to compute them without using the log-log transformation. Confidence bands can also be obtained using predictCox() by setting the argument band to TRUE. As for the predict.CauseSpecificCox() function, 10,000 simulations will be used to compute the confidence bands; this can be changed specifying the argument nsim. band. The function as. data. table.predictCox() makes it easy to extract subsets from "predictCox" object:

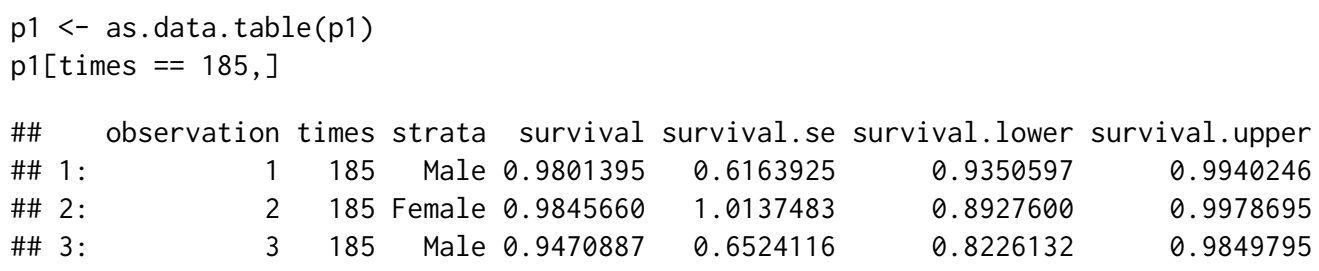

\section{Coverage of the confidence interval and the confidence bands}

To assess the validity of the estimation of the standard error, we performed a simulation study. The sample size was varied between 50 and 10000. For each sample size, 5000 datasets were simulated using the SimCompRisk () function from the riskRegression package. The time of first event typically ranged between 0.001 and 20 with a median around 4 . For each dataset, a Cox model specific to cause 1 and a 2 cause-specific Cox model were fitted considering 2 covariates (X1 and X2). The survival, absolute risk, their confidence intervals were estimated (with or without log-log transformation) at time $1,1.5,2,3,4,5,6$, and 7 conditional on $X 1=0$ and $X 2=1$. The confidence bands over those 8 times were also computed.

The true absolute risk was defined as the median of the absolute risks over the 5000 datasets. The coverage of the confidence intervals was computed as the percentage of times that the true absolute 
risk was inside the confidence interval, at a given time. The coverage of the confidence bands was computed as the percentage of times that the true absolute risk was inside the confidence bands, simultaneously for all of the 8 times.

As expected, the coverage of the confidence intervals is improving with increasing sample size and reaches its nominal level of $95 \%$ at around sample size 1000 (figure Figure 4, left panel). Using a log-log transformation leads to better small sample properties and similar large sample properties (figure Figure 4, right panel). A larger sample size was necessary for the confidence bands to converge toward the nominal coverage level ( $n=5000$, figure Figure 5 left panel). Again log-log transformation leads to better small sample properties. We only displayed here the coverage for the absolute risk but similar coverage was obtained for the survival function.

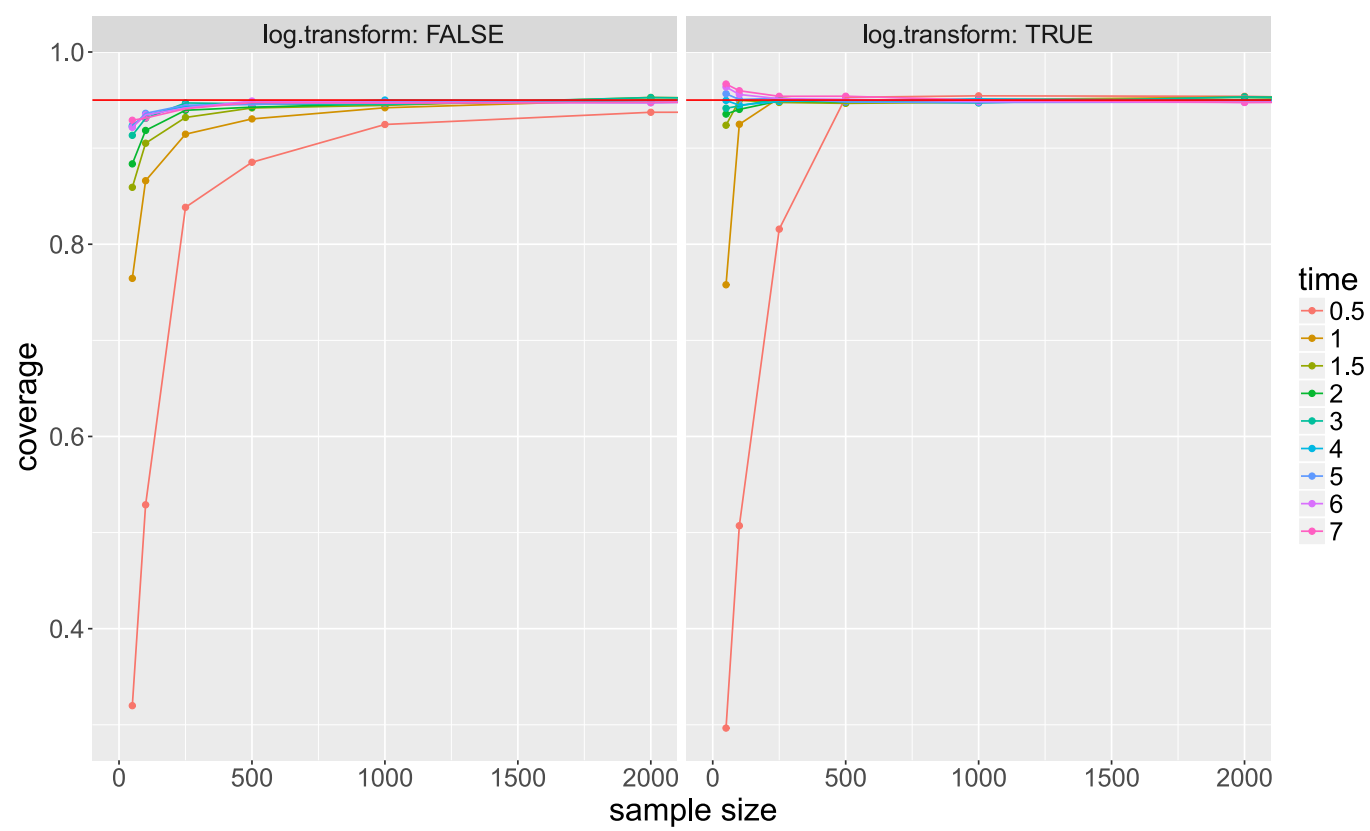

Figure 4: Coverage of the asymptotic confidence interval of the absolute risk plotted against the sample size. Each color corresponds to a prediction time. The figure is only shown for samples size below 2000 since for larger sample sizes the coverage is always approximately equal to 0.95 . Left panel: confidence intervals are computed on the original scale. Right panel: confidence intervals are computed on the log-log scale and back-transformed.

\section{Runtime and memory usage}

\section{Baseline hazard}

We compare the performance of predictCox() regarding the estimate of the baseline hazard function with that of the function survival: : basehaz(). For this purpose we simulate data with 10 covariates including both continuous and discrete type using the sampleData().

In our performance study we vary the sample size ranging from 500 to $1,000,000$ observations and consider both stratified:

Surv(time, event) $\sim \operatorname{strata}(X 1)+X 2+X 3+X 4+X 5+X 6+X 7+X 8+X 9+X 10$

and non-stratified Cox regression models:

Surv(time, event) $\sim x_{1}+x_{2}+x_{3}+x_{4}+x_{5}+x_{6}+x_{7}+x_{8}+x_{9}+x_{10}$

The computation times were estimated using the rbenchmark package (Kusnierczyk, 2012) and averaged across 100 simulated data sets. The memory usage was estimated using the profvis package (Chang and Luraschi, 2017) and averaged across 10 simulations. When the execution time of the function was extremely fast (i.e. $<0.005 \mathrm{~s}$ ), the memory usage could not be reliably assessed and was set to NA.

Memory usage and computation time are displayed on figure Figure 6 and Figure 7 . Both functions lead to a reasonable computation time $(<1 \mathrm{~min})$ even when considering very large datasets (e.g. $>10,000$ observations). Nevertheless the predictCox() function outperforms the basehaz() function by a factor 


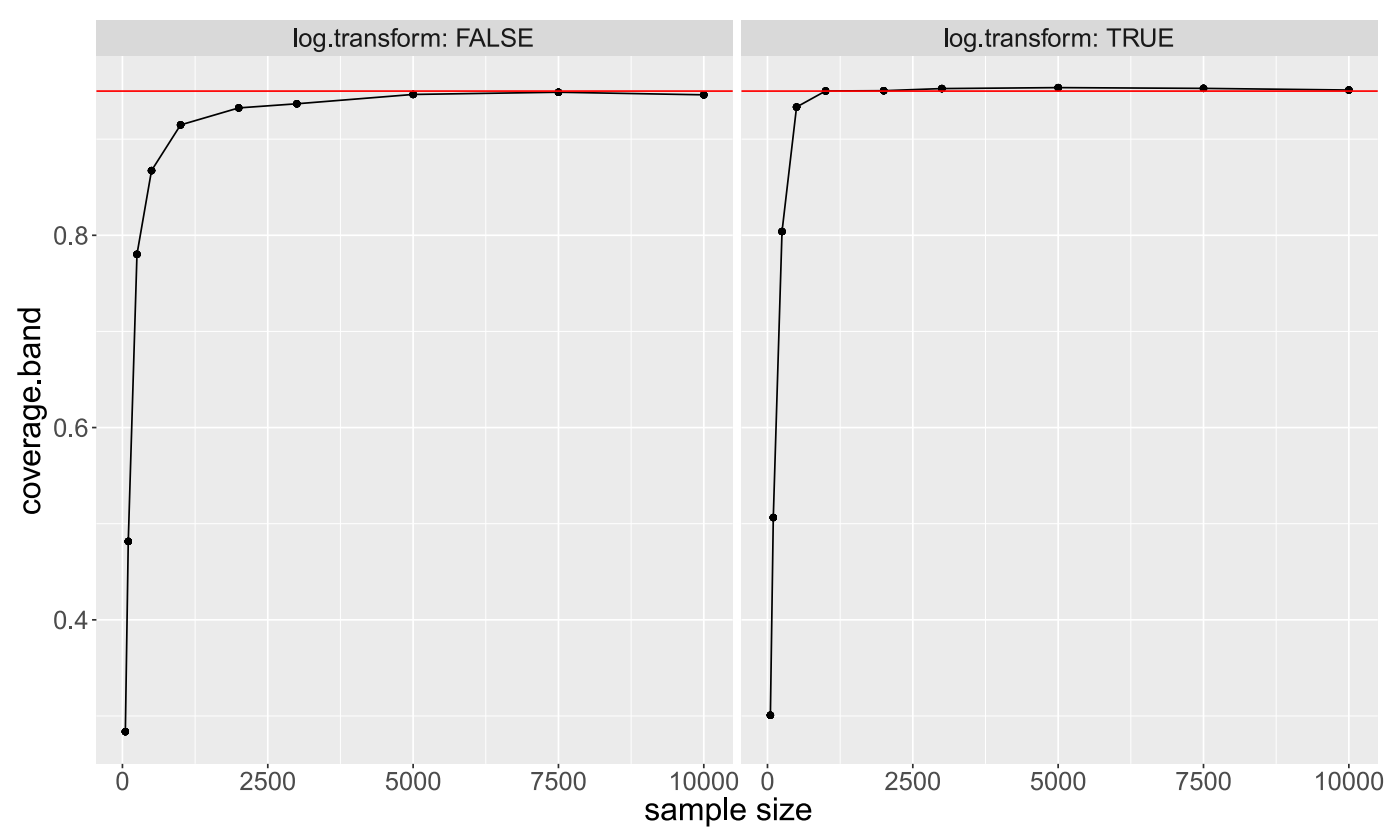

Figure 5: Coverage of the asymptotic confidence band of the absolute risk plotted against the sample size. Left panel: confidence intervals are computed on the original scale. Right panel: confidence intervals are computed on the log-log scale and back-transformed.

varying between 3 and 11 in terms of computation time. The gain in speed is especially expressed in large datasets. Memory usage is also lower for predictCox() and decreases by a factor between 1 and 1.6 as compared to basehaz().

\section{Absolute risk}

We now compare two implementations for computing the standard errors of the absolute risk. The default implementation corresponds to setting the argument store.iid to "full" when calling predict.CauseSpecificCox() or predictCox() while the second is obtained by setting store.iid to "minimal". The two implementations are described in more detail appendix B.

First we compare their computation time and memory consumption when making only one prediction. As before, we simulated datasets for $K=2$ using the SimCompRisk() function with increasing sample size. Then, the two cause-specific Cox models were fitted to each of the simulated datasets using riskRegression: : CSC. Then we measured the computation time and memory usage necessary to estimate the absolute risk with its standard error for the first observation at time 4 , when using the argument store. $\mathrm{iid="minimal"} \mathrm{or} \mathrm{store.} \mathrm{iid}=$ "full" in predict. CauseSpecificCox().

The results are show on figure Figure 8 . While both implementations lead to a similar computation time, the memory usage for store i id="minimal" grows linearly while for store. $i$ id=" full" it grows approximately in $n^{1.4}$. However, if instead of estimating the absolute risk with its standard error for one prediction, we estimate it for all the observations the implementation store. i id="minimal" becomes slower compared to store. $\mathrm{i}$ id="full" (e.g. 28.5 minutes vs. 6 minutes for $n=2000$ ). 


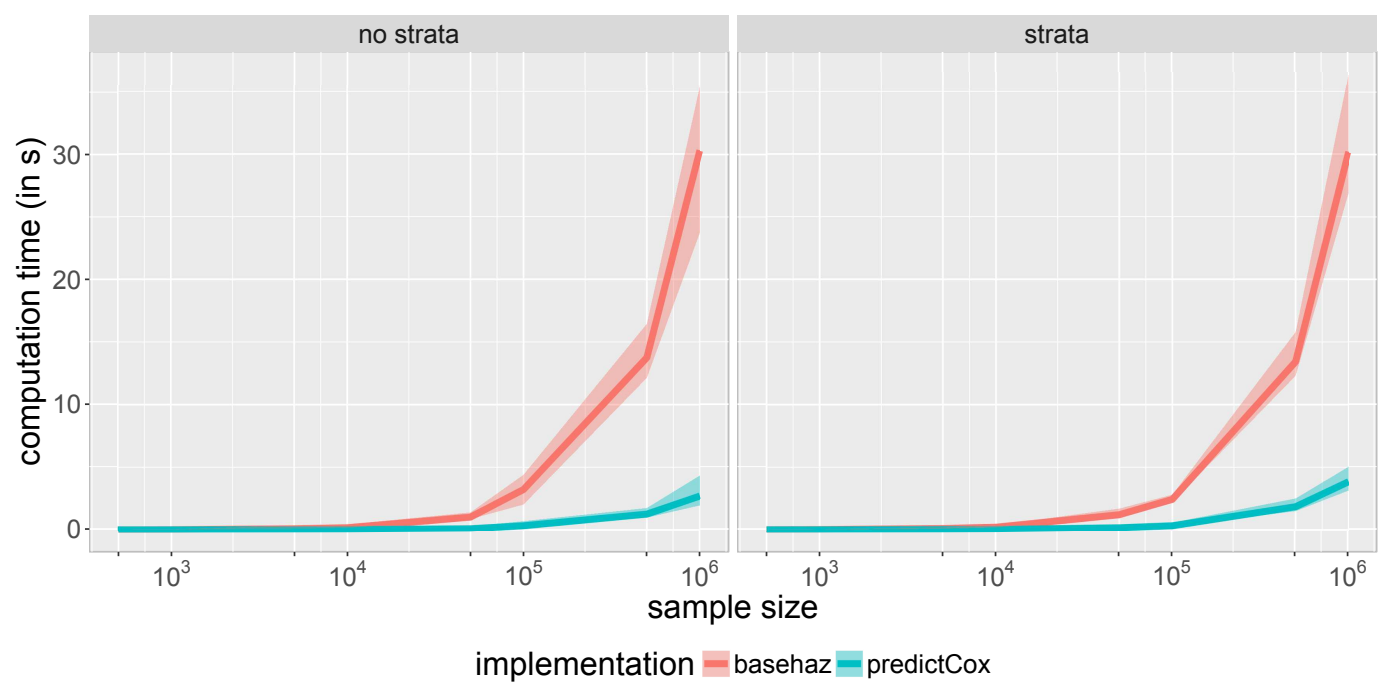

Figure 6: The computation time (in seconds) of predictCox() and basehaz() plotted against the sample size for a Cox model (left panel) and a stratified Cox model (right panel). The $\mathrm{x}$ axis is displayed using a logarithmic scale but its labels refer to the (untransformed) sample size. The curves represent the median values over 100 simulations while the shaded areas represent the $95 \%$ confidence intervals.

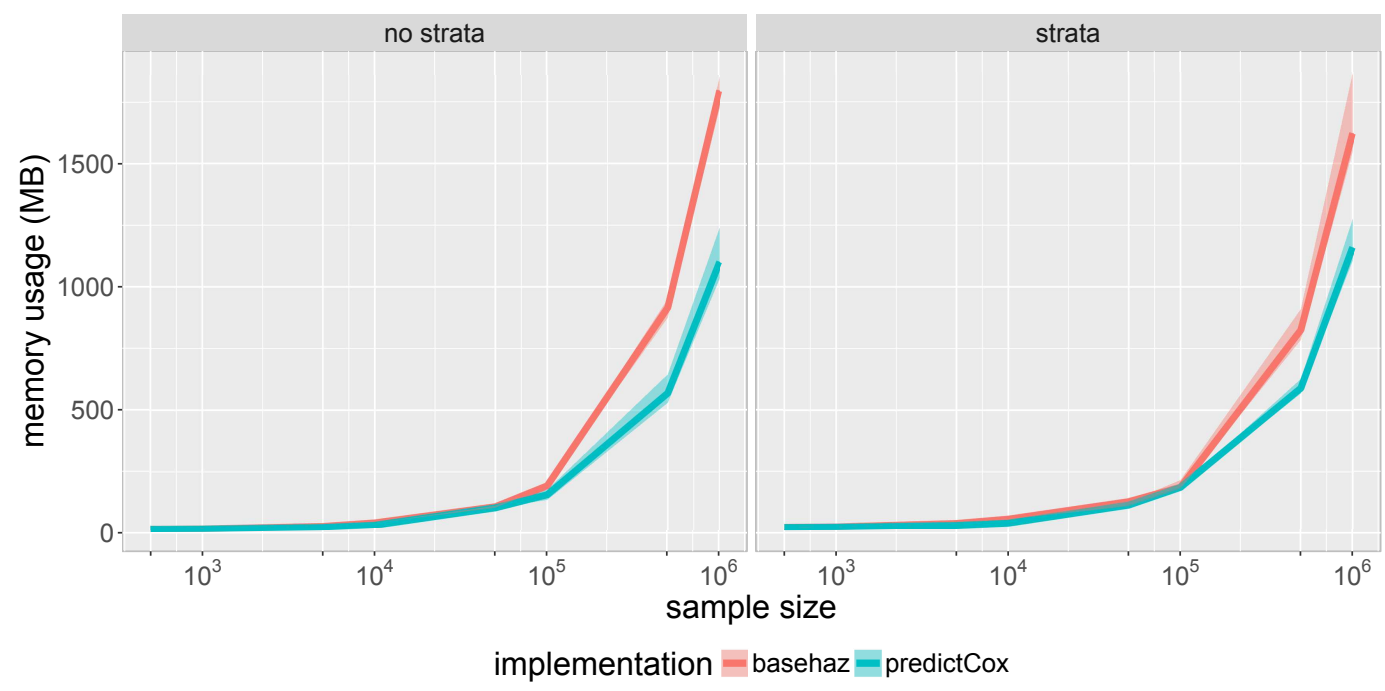

Figure 7: The memory usage (in megabytes) of predictCox() and basehaz() plotted against the sample size for a Cox model (left panel) and a stratified Cox model (right panel). The $\mathrm{x}$ axis is displayed using a logarithmic scale but its labels refer to the (untransformed) sample size. The curves represent the median values over 100 simulations while the shaded areas represent the $95 \%$ confidence intervals. 


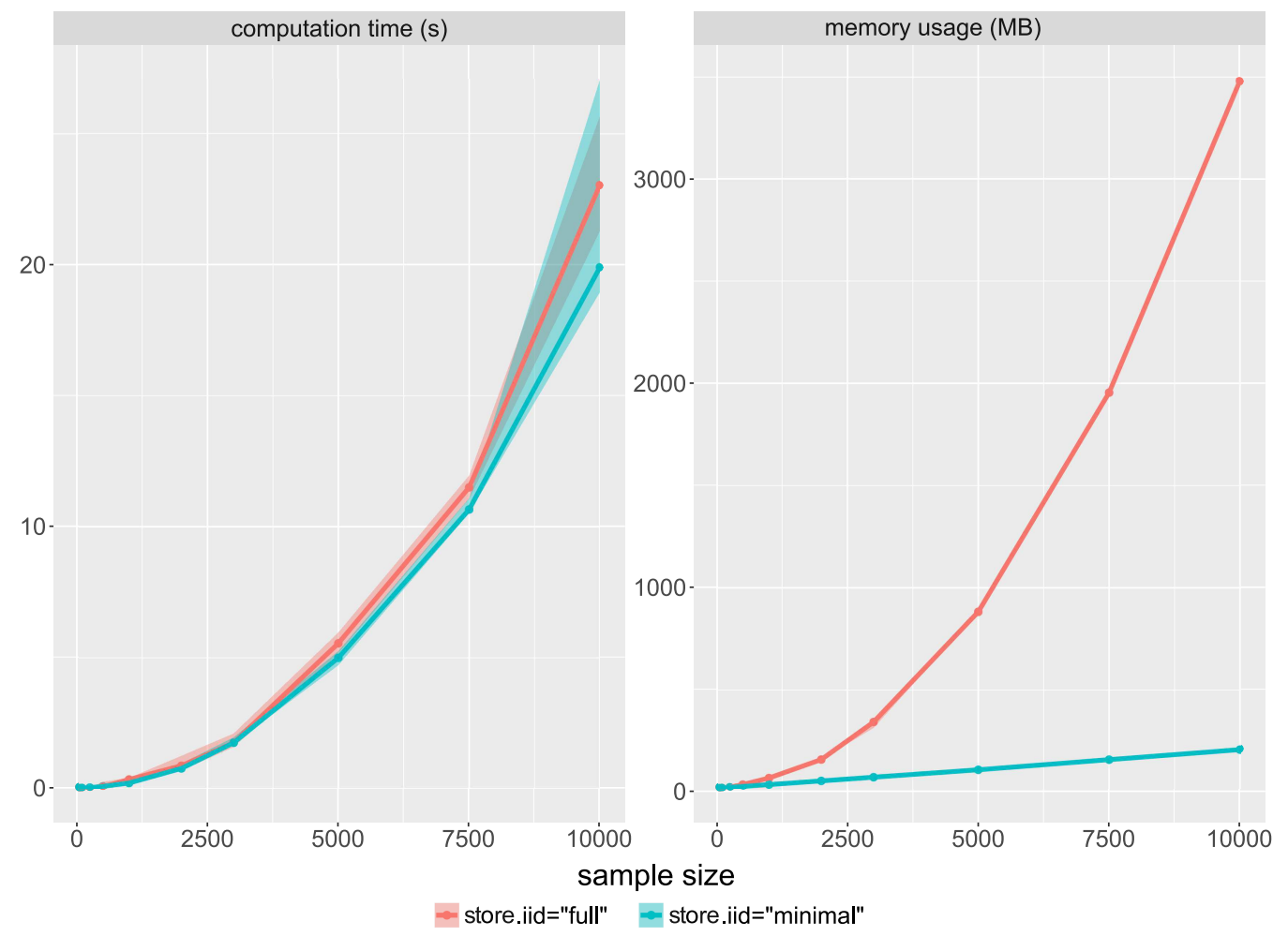

Figure 8: The computation time (left panel) and memory usage (right panel) for computing the absolute risk with its standard error for one observation plotted against the sample size, setting the argument store.iid to "full" or "minimal" when calling predict.CauseSpecificCox(). The curves represent the median values over 100 simulations while the shaded areas represent the $95 \%$ confidence intervals. 


\section{Summary}

This paper introduces new features of the riskRegression package for prediction of absolute risks from cause-specific Cox regression models using computationally efficient functions. Table 1 summarizes the main functions described in this paper. Confidence intervals and confidence bands for the absolute risks can be computed using the predict() function and displayed using the print() method. The predictCox() function can be applied on "coxph" and "cph" objects to predict the survival with its confidence interval or confidence bands. In both cases, the autoplot() function can display the predicted risk (or survival) over time. When dealing with small to moderate sample sizes, we advise to compute confidence intervals or confidence bands using a log-log transformation (argument log. transform).

Table 1: Functions implemented in the riskRegression package for making prediction from Cox regression models

\begin{tabular}{|c|c|}
\hline $\csc ()$ & Fit cause-specific Cox models \\
\hline predict() & $\begin{array}{l}\text { Predict covariate specific absolute risks for given time hori- } \\
\text { zons }\end{array}$ \\
\hline $\operatorname{iidCox}()$ & $\begin{array}{l}\text { Compute the influence function of the baseline hazard esti- } \\
\text { mator and of the partial likelihood estimator of the regres- } \\
\text { sion coefficients }\end{array}$ \\
\hline predictCox () & $\begin{array}{l}\text { Compute the (cumulative) baseline hazard function and } \\
\text { predictions of hazards and survival probabilities in new } \\
\text { data }\end{array}$ \\
\hline & Graphical display of the predicted risk across time \\
\hline
\end{tabular}

Brice Ozenne

Section of Biostatistics, Department of Public Health, University of Copenhagen

Østerfarimagsgade 5, 1014 Copenhagen

Denmark

broz@sund.ku.dk

Anne Lyngholm Sørensen

Section of Biostatistics, Department of Public Health, University of Copenhagen

Østerfarimagsgade 5, 1014 Copenhagen

Denmark

als@sund.ku.dk

Thomas Scheike

Section of Biostatistics, Department of Public Health, University of Copenhagen

Østerfarimagsgade 5, 1014 Copenhagen

Denmark

thscasund.ku.dk

Christian Torp-Pedersen

Public Health and Epidemiology Group, Department of Health Science and Technology, Aalborg University

Niels Jernes Vej 12, 9220 Aalborg

Denmark

ctp@hst.aau.dk

Thomas Alexander Gerds

Section of Biostatistics, Department of Public Health, University of Copenhagen

Østerfarimagsgade 5, 1014 Copenhagen

Denmark

tag@iostat.ku.dk

\section{Bibliography}

P. K. Andersen, Ø. Borgan, R. D. Gill, and N. Keiding. Statistical Models Based on Counting Processes. Springer Series in Statistics. Springer-Verlag, New York, 1993. URL http://dx. doi . org/10.1007/ 
978-1-4612-4348-9. [p441, 447]

J. Benichou and M. H. Gail. Estimates of absolute cause-specific risk in cohort studies. Biometrics, 46 (3):813-826, 1990. URL https://dx. doi.org/10.2307/2532098. [p440,441]

N. Breslow. Covariance analysis of censored survival data. Biometrics, 30(1):89-99, 1974. URL https://dx.doi.org/10.2307/2529620. [p449]

W. Chang and J. Luraschi. Profvis: Interactive Visualizations for Profiling $R$ Code, 2017. URL https: //CRAN. R-project. org/package=profvis. R package version 0.3.3. [p451]

S. Cheng, J. P. Fine, and L. Wei. Prediction of cumulative incidence function under the proportional hazards model. Biometrics, pages 219-228, 1998. URL https://dx. doi.org/10.2307/2534009. [p448]

D. R. Cox. Regression models and life-tables. Journal of the Royal Statistical Society B, 34(2):187-220, 1972. URL http://dx.doi.org/10.1007/978-1-4612-4380-9_37. [p441]

B. Efron. The efficiency of Cox's likelihood function for censored data. Journal of American Statistical Association, 72(359):557-565, 1977. URL https://dx. doi.org/10.2307/2286217. [p449]

J. P. Fine and R. J. Gray. A proportional hazards model for the subdistribution of a competing risk. Journal of the American Statistical Association, 94(446):496-509, 1999. URL https://dx. doi .org/10. 1080/01621459.1999.10474144. [p440]

T. Gerds and M. Schumacher. On functional misspecification of covariates in the Cox regression model, 2001. ISSN 0006-3444. URL https://dx. doi.org/10.1093/biomet/88.2.572. [p447]

T. A. Gerds, T. H. Scheike, and P. K. Andersen. Absolute risk regression for competing risks: Interpretation, link functions, and prediction. Statistics in Medicine, 31(29):3921-3930, 2012. URL https://dx.doi.org/10.1002/sim. 5459. [p440]

F. E. Harrell Jr. Rms: Regression Modeling Strategies, 2017. URL https: //CRAN. R-project. org/package= rms. R package version 5.1-1. [p440]

K. K. Holst and T. Scheike. Mets: Analysis of Multivariate Event Times, 2017. URL https://CRAN. Rproject. org/package=mets. R package version 1.2.2. [p457]

W. Kusnierczyk. Rbenchmark: Benchmarking Routine for R, 2012. URL https: //CRAN. R-project.org/ package=rbenchmark. R package version 1.0.0. [p451]

H. Putter, L. de Wreede, M. Fiocco, and with contributions by Ronald Geskus. Mstate: Data Preparation, Estimation and Prediction in Multi-State Models, 2016. URL https://CRAN. R-project.org/package= mstate. R package version 0.2.10. [p440]

N. Reid. Influence functions for censored data. The Annals of Statistics, 9(1):78-92, 1981. URL http://dx.doi.org/10.1214/aos/1176345334. [p447]

T. H. Scheike and Y. Sun. Maximum likelihood estimation for tied survival data under Cox regression model via EM-algorithm. Lifetime Data Analysis, 13:399-420, 2007. URL https://dx. doi . org/10. 1007/s10985-007-9043-3. [p449]

T. H. Scheike and M.-J. Zhang. Flexible competing risks regression modeling and goodness-of-fit. Lifetime data analysis, 14(4):464-483, 2008. URL http: // dx. doi . org/10. 1007/s10985-008-9094-0. [p448]

T. H. Scheike, M. J. Zhang, and T. A. Gerds. Predicting cumulative incidence probability by direct binomial regression. Biometrika, 95(1):205-220, 2008. URL https://dx. doi . org/10.1093/biomet/ asm096. [p440]

T. M. Therneau. Survival: Survival Analysis, 2017. URL https://CRAN.R-project.org/package= survival. R package version 2.41-3. [p440]

T. M. Therneau and P. M. Grambsch. Modeling Survival Data: Extending the Cox Model. Springer-Verlag, 2000. URL https://dx. doi.org/10.1007/978-1-4757-3294-8. [p449]

A. van der Vaart. Asymptotic Statistics. Cambridge University Press, 1998. URL http://dx. doi .org/ 10.1017/CB09780511802256. [p446] 


\section{Appendix A Modularity}

The $\operatorname{CSC}()$ function requires a routine to estimate the regression coefficients of the Cox model. By default, $\csc ()$ calls the $\operatorname{coxph}()$ function from the survival package to do so. This has several reasons: $\operatorname{coxph}()$ has been thoroughly tested, is reasonably fast, widely used and provides flexible modeling options. However in specific contexts, other routines may be more appropriate, e.g. faster. Also, developers that have implemented their own routine may be interested in computing the baseline hazard or the influence function.

To be able to apply the predictCox() and iidCox() functions on new classes, one needs to define the methods extracting the necessary information from the new class. For instance, the mets package (Holst and Scheike, 2017) contains an efficient routine for estimating Cox models. However "phreg" object that are quite different from "coxph" objects:

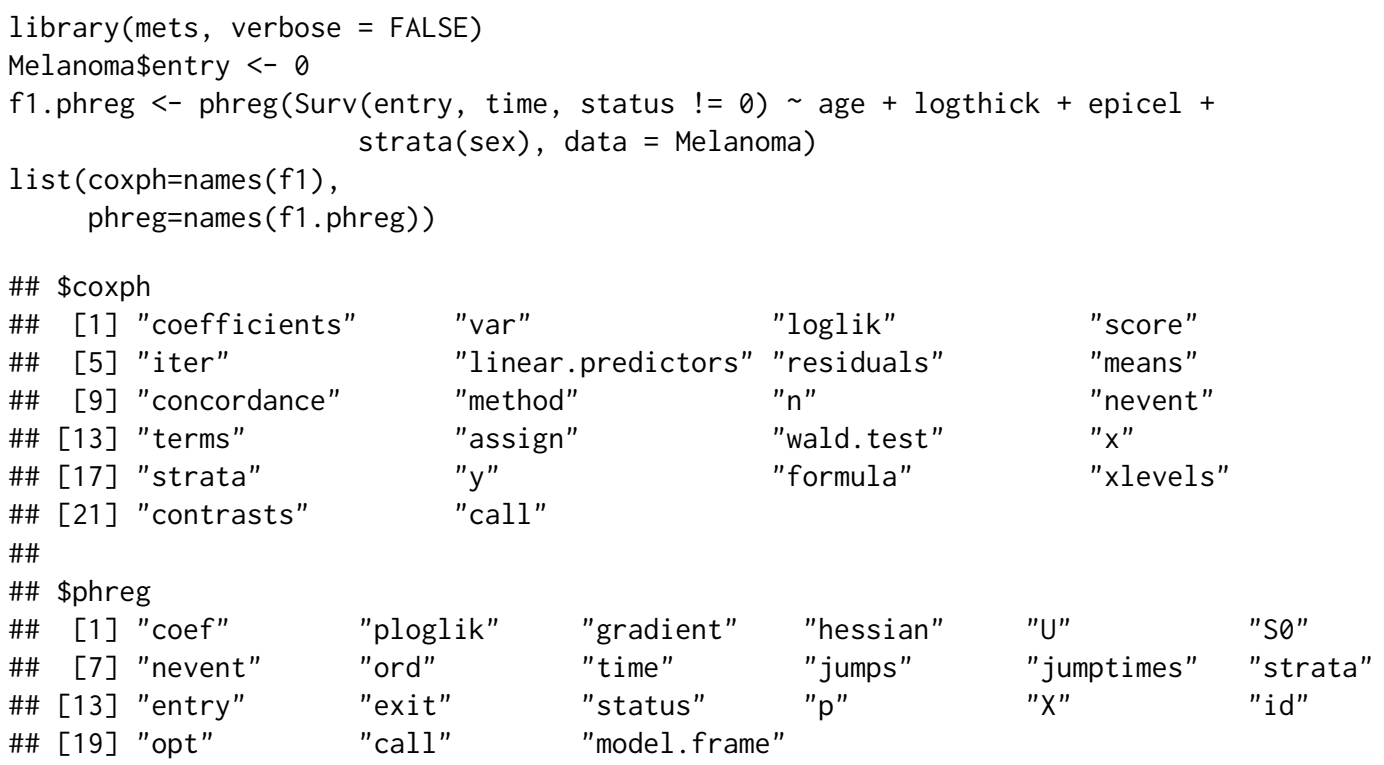

Therefore to be able to use the predictCox() and iidCox() functions on "phreg" one needs to define methods to extract:

The values used to center the covariates (CoxCenter ( ) method). Instead of working on $X$, many routines estimating the Cox model parameters works on a centered version $\tilde{X}=X-\bar{X}$. The CoxCenter() method returns $\bar{X}$ :

riskRegression: : : coxCenter. $\operatorname{coxph}(f 1)$

\#\# age logthick epicelpresent

\#\# $52.4634146 \quad 0.6181706 \quad 0.4341463$

The design matrix used to fit the Cox model (CoxDesign() method). The first two columns describe the beginning and the end of the interval of time when the individual was followed. The third contains the event type ( 0 corresponding to censoring and 1 to an observed event, e.g. death). The remaining columns contain the design matrix corresponding to the coefficients $\beta$ of the Cox model and the strata variable (if any):

head(riskRegression: : : coxDesign. coxph(f1))

\begin{tabular}{|c|c|c|c|c|c|c|c|}
\hline \#\# & start & stop & status & age & logthick & epicelpresent & strata \\
\hline \#\# 1 & 0 & 10 & 1 & 76 & 1.9110229 & 1 & 2 \\
\hline \#\# 2 & 0 & 30 & 1 & 56 & -0.4307829 & 0 & \\
\hline \#\# 3 & 0 & 35 & 0 & 41 & 0.2926696 & 0 & \\
\hline \#\# 4 & 0 & 99 & 1 & 71 & 1.0647107 & 0 & \\
\hline \#\# 5 & 0 & 185 & 1 & 52 & 2.4915512 & 1 & \\
\hline \#\# 6 & 0 & 204 & 1 & 28 & 1.5769147 & 0 & \\
\hline
\end{tabular}

The formula of the Cox model (CoxFormula() method):

riskRegression: : : coxFormula.coxph(f1) 
Surv(time, status $!=0) \sim$ age + logthick + epicel + strata(sex $)$

The value of the linear predictor $X \beta(\operatorname{CoxLP}()$ method). This function has three arguments: object, data, and center.

head $($ riskRegression: $: \operatorname{coxLP} \cdot \operatorname{coxph}(\mathrm{f} 1$, data $=$ NULL, center $=\mathrm{FALSE}))$

$\begin{array}{llllllll}\text { \#\# [1] } & 2.433793 & 1.136828 & 1.168604 & 2.332216 & 2.165533 & 1.559629\end{array}$

When setting data to NULL, the $\operatorname{CoxLP}()$ method will return the linear predictor computed on the dataset used to fit the Cox model. The center argument indicates whether the covariates should be centered before computing the linear predictor.

The number of observations used to fit the Cox model (CoxN() method):

riskRegression: : : $\operatorname{coxN} . \operatorname{coxph}(f 1)$

\#\#[1] 205

The character string indicating the strata variable(s) in the formula (CoxSpecialstrata() method):

riskRegression: : : coxSpecialStrata. $\operatorname{coxph}(\mathrm{f} 1)$

\#\# [1] "strata"

The variable encoding to which strata belongs each observation (CoxStrata() method). This variable must be univariate, aggregating all the strata variables.

head(riskRegression: : :coxStrata. $\operatorname{coxph}(\mathrm{f1}$, data $=$ NULL, strata.vars = "strata(sex)"))

\#\# [1] Male Male Male Female Male Male

\#\# Levels: Female Male

Similarly to $\operatorname{CoxLP}()$, when setting data to NULL, the CoxStrata() method will return the strata variable computed on the dataset used to fit the Cox model.

The variance-covariance matrix of the regression coefficients ( $\operatorname{Cox} V \operatorname{arCov}()$ method):

riskRegression: : : coxVarCov. $\operatorname{coxph}(f 1)$

$\begin{array}{lrrr}\text { \#\# } & \text { age } & \text { logthick epicelpresent } \\ \text { \#\# age } & 6.553939 \mathrm{e}-05 & -0.0002102408 & -0.0004363469 \\ \text { \#\# logthick } & -2.102408 \mathrm{e}-04 & 0.0206977373 & 0.0076882093 \\ \text { \#\# epicelpresent } & -4.363469 \mathrm{e}-04 & 0.0076882093 & 0.0692047486\end{array}$

Most of the above methods correspond to a very small piece of code that reformats the information contained in the object, e.g.:

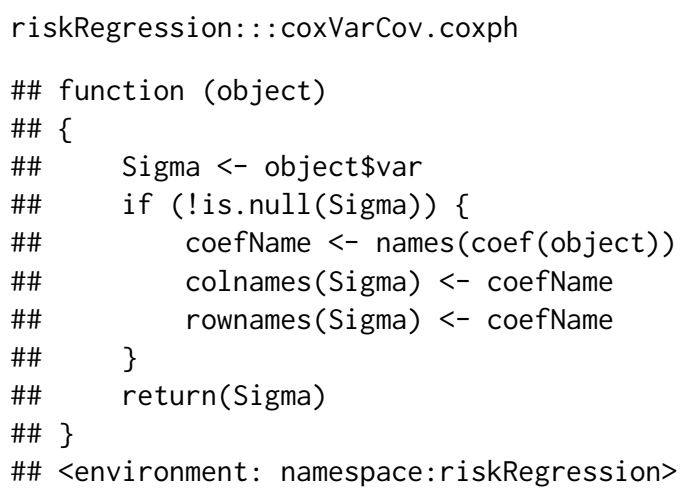

We refer to the help page of each method for a more precise description of each method arguments and expected output, as well as examples. Once all of the methods have been defined for a new object (e.g. "phreg"), predictCox() and iidCox() can be applied on the new object:

all.equal(predictCox(f1), predictCox (f1.phreg))

\#\# [1] TRUE

all. equal(iidCox(f1), iidCox(f1.phreg))

\#\# [1] TRUE 


\section{Appendix B Saving the influence functions}

The function iidCox () computes the estimates of the influence functions $\phi_{\beta_{j}}, \phi_{\lambda_{0 j}}$, and $\phi_{\Lambda_{0 j}}$ for a given set of covariates and time points:

f1.iid <- iidCox(f1)

The default implementation (store.iid="full") stores the influence function for the baseline cumulative hazard as a list of matrices, one for each strata. The size of each matrix is the number of observations $n$ times the number of unique event times $n_{T}(z)$ in stratum $z$. Storing the influence function can be very memory demanding when considering large datasets. This is why the influence function is only temporary stored during the execution of the predict () method.

When dealing with very large datasets, e.g. following $n=20000$ patients during $n_{T}=365$ days, storing the influence function can be too memory demanding. Instead of computing and storing $\phi_{\lambda_{0 j}}$, an alternative solution is to only store the necessary quantities to compute $\phi_{\lambda_{0 j}}$ :

$$
\frac{1\left\{\tilde{T}_{i}=t, \tilde{D}_{i}=j\right\}}{\widehat{\mathcal{S}^{(0)}}\left(\tilde{T}_{i}, \hat{\beta}_{j}, Z_{i}\right)}, E\left(t, \hat{\beta}_{j}, z\right) \hat{\lambda}_{0 j, z}(t), \int_{0}^{t} E\left(s, \hat{\beta}_{j}, z\right) \hat{\lambda}_{0 j, z}(s) \mathrm{d} s, \frac{\hat{\lambda}_{0 j, z}(t)}{\widehat{\mathcal{S}^{(0)}\left(t, \hat{\beta}_{j}, z\right)}}, \int_{0}^{t} \frac{\hat{\lambda}_{0 j, z}(s)}{\widehat{\mathcal{S}^{(0)}\left(s, \hat{\beta}_{j}, z\right)}} d s .
$$

These quantities are lists containing $L$ vectors of length $n$ or $n_{T}(z)$. Since in most applications $n$ and $n_{T}(z)$ are large compared to $L$ this approach is much more memory efficient. Setting the argument store. iid to "minimal" when calling i idCox will return the influence function using this alternative storage method:

f2.iid <- iidCox(f1, store.iid = "minimal")

We can compare the memory cost of the default implementation vs. the alternative one:

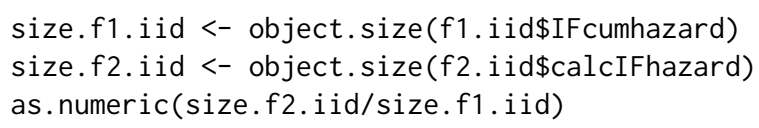

\#\# [1] 0.08627399

and see that the memory use for storing the influence function of the cumulative hazard has been divided by more than 10 .

Computing the influence function of the absolute risk with the default implementation only requires to:

- use $\widehat{\phi}_{\Lambda_{0 j, z}}\left(\mathcal{X}_{i} ; t\right)$ and $d \widehat{\phi}_{\Lambda_{0 j, z}}\left(\mathcal{X}_{i} ; t\right)$ with formula (12) and (13) to obtain $\hat{\phi}_{\Lambda_{j, z}}\left(\mathcal{X}_{i} ; t, x\right)$ and $d \hat{\phi}_{\Lambda_{j, z}}\left(\mathcal{X}_{i} ; t, x\right)$.

- use formula (14) to obain $\widehat{\phi}_{F_{1}}\left(\mathcal{X}_{i}, t, x, z\right)$.

When using the alternative implementation, $\widehat{\phi}_{\Lambda_{0 j, z}}\left(\mathcal{X}_{i} ; t\right)$ and $d \widehat{\phi}_{\Lambda_{0 j, z}}\left(\mathcal{X}_{i} ; t\right)$ have not been computed. Therefore an additional step is needed:

- use (16) with formula (10) and (11) to compute $\widehat{\phi}_{\Lambda_{0 j, z}}\left(\mathcal{X}_{i} ; t\right)$ and $d \widehat{\phi}_{\Lambda_{0 j, z}}\left(\mathcal{X}_{i} ; t\right)$.

The two implementations will lead to the same influence function and therefore the same confidence intervals or confidence bands.

The alternative implementation is performed iterating over the set of covariates used to make the predictions, avoiding to store the influence function of the baseline hazard for all event times and strata. It will also not compute the influence function at unnecessary times and strata. Thus it should always be more memory efficient and, when asking for a single prediction, it should also be have a lower computation time. However, compared to the default implementation where $\widehat{\phi}_{\Lambda_{0 j, z}}\left(\mathcal{X}_{i} ; t\right)$ and $d \widehat{\phi}_{\Lambda_{0 j, z}}\left(\mathcal{X}_{i} ; t\right)$ are only computed once, the alternative implementation recomputes these quantities for each prediction.

Therefore when the prediction is to be made for many different sets of covariates (i.e. new patients) this may lead to a substantial increase in computation time. See the subsection Runtime and memory usage for more details.

To use the implementation for computing the standard errors, confidence intervals, or confidence bands one must set the argument store. iid to "minimal":

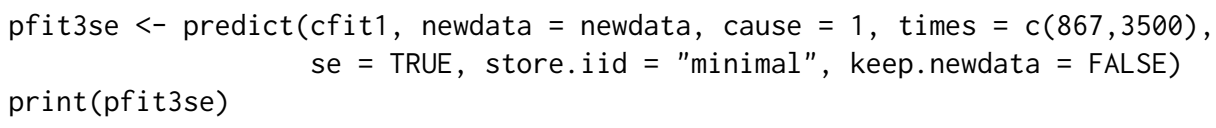




\begin{tabular}{|c|c|c|c|c|c|c|c|}
\hline \#\# & observation & times & strata & absRisk & absRisk.se & absRisk.lower & absRisk.upper \\
\hline \#\# 1: & 1 & 867 & sex=Female & 0.021 & 0.122 & 0.00738 & 0.0478 \\
\hline \#\# 2: & 2 & 867 & sex=Male & 0.149 & 0.161 & 0.07356 & 0.2502 \\
\hline \#\# 3: & 1 & 3500 & sex=Female & 0.117 & 0.151 & 0.05552 & 0.2025 \\
\hline \#\# 4: & 2 & 3500 & sex $=$ Male & 0.428 & 0.320 & 0.20416 & 0.6355 \\
\hline
\end{tabular}

range (pfit3se\$absRisk. se-pfit1se\$absRisk.se)

range (pfit3se\$absRisk. upper-pfit1se\$absRisk. upper)

range (pfit3se\$absRisk. lower-pfit1se\$absRisk. lower)

\#\# [1] $-2.775558 \mathrm{e}-17 \quad 5.551115 \mathrm{e}-17$

\#\# [1] $-5.551115 \mathrm{e}-17 \quad 0.000000 \mathrm{e}+00$

\#\# [1] $-5.551115 \mathrm{e}-17 \quad 0.000000 \mathrm{e}+00$

This option also applies when computing standard errors, confidence intervals, or confidence bands for the survival probabilities:

p2 $<-\operatorname{predictCox}(f 1$, newdata $=\operatorname{Melanoma}[3: 5$,$] ,$ times $=c($ Melanomatime $[5: 7], 1000)$, se $=$ TRUE, store.iid = "minimal", type="survival")

p2 <- as.data. table(p2)

$\mathrm{p} 2[$ times $==185$, survival. lower]-p1[times $==185$, survival.lower]

$\mathrm{p} 2[$ times $==185$, survival.upper] $-\mathrm{p} 1[$ times $==185$, survival.upper $]$

\#\# [1] $0.000000 \mathrm{e}+00 \quad 1.110223 \mathrm{e}-16-1.110223 \mathrm{e}-16$

\#\# [1] 0000 